\title{
Sodium Glucose Co-Transporter 2 (SGLT2) Inhibitors in Type 2 Diabetes: A Literature Review of Approved Products
}

\author{
Lucio R. Volino1,2, Eva Y. Pan1, Rupal Patel Mansukhani1,3 \\ ${ }^{1}$ Department of Pharmacy Practice and Administration, Ernest Mario School of Pharmacy, Rutgers, The State \\ University of New Jersey, Piscataway, USA \\ ${ }^{2}$ The Great Atlantic and Pacific Tea Company, Kenilworth, USA \\ ${ }^{3}$ Morristown Medical Center, Morristown, USA \\ Email: volinol@pharmacy.rutgers.edu
}

Received 30 August 2014; revised 30 September 2014; accepted 16 October 2014

Copyright (C) 2014 by authors and Scientific Research Publishing Inc. This work is licensed under the Creative Commons Attribution International License (CC BY). http://creativecommons.org/licenses/by/4.0/

\section{(c) (i) Open Access}

\begin{abstract}
Diabetes mellitus continues to be a major health issue worldwide. Despite all of the treatment options available on the market, many patients with diabetes fail to reach their treatment goals. Novel agents such as the Sodium-Glucose Cotransporter 2 (SGLT2) inhibitors show promise in effectively lowering blood glucose. Objective: To review the scientific literature for efficacy information regarding the use of approved SGLT2 inhibitors (canagliflozin, dapagliflozin and empagliflozin) in the treatment of Type 2 Diabetes Mellitus (T2DM). Methods: A MEDLINE (1950-August 2014) literature review was performed. All of the literature published as an original clinical trial was included in this review. Other pertinent articles published related to the original clinical trial were also included. Meta-analysis type studies were not selected for this review. Conclusions: With an increasing prevalence and incidence of type 2 diabetes mellitus worldwide, there is an apparent need for effective therapeutic strategies to combat this chronic and progressive disease. SGLT2 inhibitors offer this potential. Recently approved agents (canagliflozin, dapagliflozin and empagliflozin) have shown significant promise as mono- and add-on therapy to current glucose-lowering regimens that may not otherwise be providing sufficient glycemic control in T2DM patients.
\end{abstract}

\section{Keywords}

Canagliflozin, Dapagliflozin, SGLT2 Inhibitors, Type 2 Diabetes Mellitus, Empagliflozin 


\section{Introduction}

Diabetes mellitus continues to be a major health issue worldwide, affecting nearly 26 million adults in the United States. Controlling blood glucose levels is essential in managing symptoms and preventing complications associated with the disease. In 2011, close to $85 \%$ of US adults with diabetes reported taking antihyperglycemic medication [1].

Despite all the treatment options available on the market, many patients with diabetes fail to reach their treatment goals. Most of these medications depend on the presence or action of insulin to exert their therapeutic effect. This can provide little benefit to patients whose disease progression has led to deterioration in pancreatic beta cell function. Additionally, these agents are associated with concerning side effects, including the risk of inducing hypoglycemia [2]-[4].

A new class of agents has emerged with glycemic control via alternate means, specifically by inhibiting the reabsorption of glucose and increasing its excretion from the kidneys. This novel approach of the Sodium-Glucose Cotransporter 2 (SGLT2) inhibitors shows promise in effectively lowering blood glucose in a noninsulindependent way. Sodium-Glucose Cotransporter 2 inhibitors exert their effect by enhancing renal glycosuria. The extent to which they induce glycosuria is dependent on the plasma glucose concentration. As a result, blood glucose levels cannot be lowered below physiological levels and hence, the risk of hypoglycemia is not concerning [5]. This mechanism of action allows use as monotherapy or in combination with current antidiabetes medications, including insulin therapy [2]-[4]. Sodium-Glucose Cotransporter 2 inhibitors have also been shown to promote weight loss at the same time, either independently or in combination with other antidiabetic agents. However they have a risk of potentially increasing the likelihood of genitourinary tract infections [6].

The United States Food and Drug Administration (FDA) recently approved three SGLT2 inhibitors, canagliflozin in 2013, dapagliflozin and empagliflozin in 2014. This article discusses the SGLT2 inhibitors as new approaches to managing type 2 diabetes mellitus (T2DM), focusing on the evidence available regarding the efficacy and safety of this emerging class of antidiabetic agents. Canagliflozin, dapagliflozin, and empagliflozin were selected for this review since they are the only agents currently approved by the FDA.

\section{Data Sources}

Figure 1 illustrates the literature search and selection process details used in the identification of clinical trials for this review. A literature review was performed in MEDLINE (1950-August 2014) using the keywords diabetes mellitus type 2 AND canagliflozin OR dapagliflozin OR empagliflozin. The references identified from the literature review were then evaluated. All of the literature retrieved from MEDLINE that was published as an original clinical trial was included in this review. Other pertinent articles published related to the original clinical trials were also considered. Meta-analysis type studies were not selected for this review. References included in this review were limited to studies conducted in humans and written in the English language.

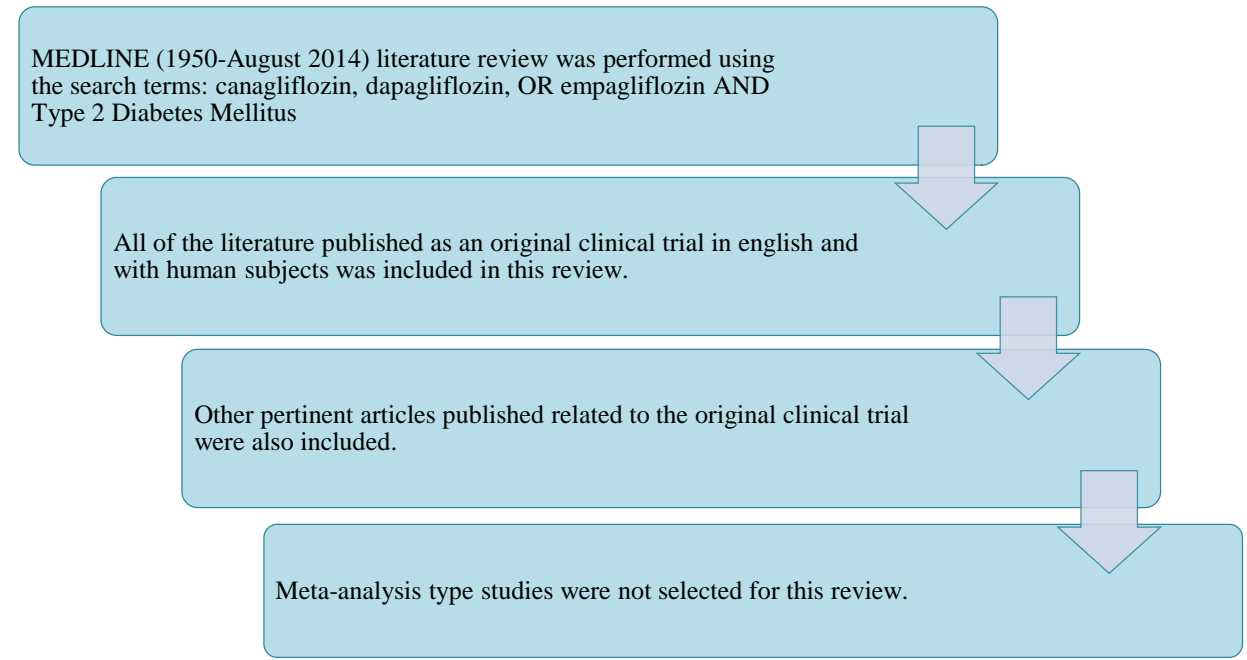

Figure 1. Literature search and clinical trial selection details. 


\section{SGLT2 Product Review Summaries}

\subsection{Canagliflozin}

Canagliflozin, (1S)-1,5-anhydro-1-[3-[[5-(4-fluorophenyl)-2-thienyl]methyl]-4-methylphenyl]-D-glucitol hemihydrate, the first SGLT2 inhibitor approved in the United States, has an oral bioavailability of $65 \%$, which remains the same with or without food. However, it is recommended to be taken before the first meal of the day due to its mechanism of reducing postprandial glucose excursions. Peak plasma concentrations are reached within one to two hours post-dose with a terminal half-life of 10.6 hours and 13.1 hours for the respective 100 $\mathrm{mg}$ and $300 \mathrm{mg}$ doses. Canagliflozin exhibits extensive protein binding (99\%), mainly to albumin, which does not affect plasma concentrations. It is metabolized primarily through $O$-glucuronidation and marginally through CYP3A4 (7\%), and is excreted through fecal and renal routes. Though renal impairment may lead to a change in maximum plasma concentration $\left(\mathrm{C}_{\max }\right)$ and area under the curve (AUC), these changes are not clinically relevant. However, since canagliflozin works by reducing glucose reabsorption in the kidney, pharmacodynamic response to the drug declines as the severity of renal impairment increases. Therefore, it is contraindicated in severe renal impairment, end stage renal disease, or patients on dialysis. According to Child-Pugh class grading, mild and moderate hepatic impairment do not warrant dose adjustments with canagliflozin [4].

The efficacy of canagliflozin was studied in ten trials ranging from 12 to 52 weeks and at doses of $50 \mathrm{mg}, 100$ mg, $200 \mathrm{mg}$, and $300 \mathrm{mg}$ (Table 1) [7]-[16]. The greatest difference in hemoglobin A1C (HbA1C) (\%) reduction compared to placebo was $-1.16 \%$ with canagliflozin $300 \mathrm{mg}$ after 26 weeks [13]. Eight out of ten studies reported that HbA1C (\%) reductions were statistically significant [7] [8] [10] [11] [13]-[16]. All ten trials found that a greater percentage of patients reached the HbA1C (\%) goal of $<7.0 \%$ with canagliflozin treatment than with other treatments [7]-[16]. The majority of these studies also reported a statistically significant difference [7] [8] [11] [13] [14] [16].

Reductions in 2-hour postprandial glucose (2-h PPG) [10]-[14] and fasting plasma glucose (FPG) [7]-[16] were also greater in canagliflozin groups, with mean decreases in FPG ranging from $-38.3 \mathrm{mg} / \mathrm{dL}$ [10] to -11.7 $\mathrm{mg} / \mathrm{dL}$ [15] in two different canagliflozin $300 \mathrm{mg}$ groups. Since other oral antidiabetic agents are often associated with weight gain, studies also assessed the change in body weight with canagliflozin. Most studies showed a statistically significant difference with lowering body weight in the canagliflozin groups compared to other treatment groups [7]-[14] [16]. Some evidence shows a slight, yet significant reduction in systolic blood pressure by canagliflozin compared to placebo (reduction of range of -8.1 to $-3.5 \mathrm{mmHg}$ ) [8] [10] [13] [16] and sitagliptin (reduction range of -5.9 to $-2.9 \mathrm{mmHg}$ ) [11] [12].

The overall incidence of adverse events was similar between canagliflozin and control group treatment, however more patients withdrew related to canagliflozin adverse events. Since the SGLT2 inhibitors work by increasing the amount of glucose in the urine, there is a risk of urinary and genital tract infections unique to this class. Adverse events such as pollakiuria, polyuria, and volume-related effects, including postural dizziness and orthostatic hypotension, were more common in canagliflozin groups [7]-[16].

As expected, based on the mechanism of action of SGLT2 inhibitors, the majority of studies reported low incidences of hypoglycemia in canagliflozin groups which were similar to sitagliptin [11] [12] [16] and placebo [7] [8] [10] [13] [16] groups. Canagliflozin was reported to have higher rates of hypoglycemia compared to placebo when combined with other hypoglycemia-associated medications such as insulin [15] or a sulfonylurea [14] [15]. Hypoglycemia occurred significantly less in canagliflozin groups (5\% - 6\%) compared to the glimepiride treatment group (34\%) [9].

\subsection{Dapagliflozin}

Dapagliflozin, D-glucitol, 1,5-anhydro-1-C-[4-chloro-3-[(4-ethoxyphenyl)methyl]phenyl]-, (1S)-, compounded with (2S)-1,2-propanediol, hydrate (1:1:1) [2], was rejected by the FDA in January 2012 due to concerns about the cancer risk seen in studies [17]. However, after reviewing more data on its safety profile, dapagliflozin was approved by the FDA in early 2014. The pharmacokinetic and pharmacodynamic properties of dapagliflozin are similar to canagliflozin. Dapagliflozin can be administered without regard to food. The oral bioavailability of dapagliflozin $10 \mathrm{mg}$ is $78 \%$. Maximum plasma concentration is usually reached within two hours in a fasting state and administration with a high-fat meal decreases the $\mathrm{C}_{\max }$ by up to $50 \%$ without altering the AUC, but is not clinically significant. The terminal half-life is approximately 12.9 hours following a dose of $10 \mathrm{mg}$. Dapag- 
Table 1. Canagliflozin trials.

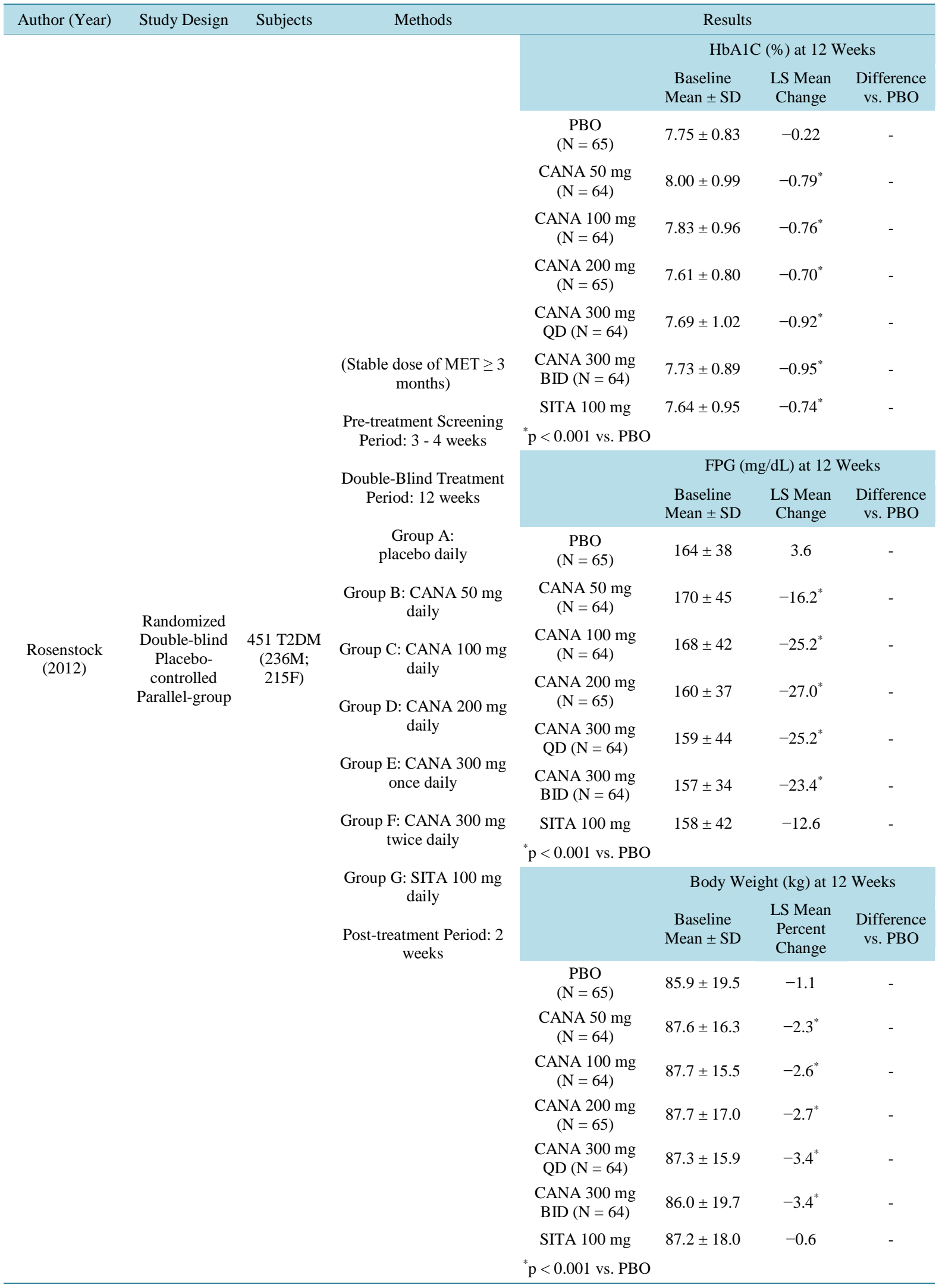




\section{Continued}

\begin{tabular}{|c|c|c|c|c|c|c|c|}
\hline \multirow{18}{*}{$\begin{array}{l}\text { Bode } \\
(2013)\end{array}$} & \multirow{18}{*}{$\begin{array}{l}\text { Randomized } \\
\text { Double-blind } \\
\text { Placebo- } \\
\text { controlled }\end{array}$} & \multirow{18}{*}{$\begin{array}{l}714 \text { T2DM } \\
\text { with } \\
\text { (396M; } \\
318 \mathrm{~F})\end{array}$} & & & \multicolumn{3}{|c|}{ HbA1C (\%) at 26 Weeks } \\
\hline & & & & & $\begin{array}{l}\text { Baseline } \\
\text { Mean } \pm \text { SD }\end{array}$ & $\begin{array}{l}\text { Adjusted } \\
\text { Mean } \\
\text { Change }\end{array}$ & $\begin{array}{l}\text { Difference } \\
\text { vs. PBO }\end{array}$ \\
\hline & & & & $\begin{array}{c}\text { PBO } \\
(\mathrm{N}=237)\end{array}$ & $7.8 \pm 0.8$ & -0.03 & - \\
\hline & & & & $\begin{array}{l}\text { CANA } 100 \mathrm{mg} \\
\quad(\mathrm{N}=241)\end{array}$ & $7.8 \pm 0.8$ & -0.6 & $-0.57^{*}$ \\
\hline & & & Single-blind, Placebo & $\begin{array}{l}\text { CANA } 300 \mathrm{mg} \\
\quad(\mathrm{N}=236)\end{array}$ & $7.7 \pm 0.8$ & -0.73 & $-0.70^{*}$ \\
\hline & & & Run-in Period: 2 weeks & ${ }^{*} \mathrm{p}<0.001$ vs. $\mathrm{PBC}$ & & & \\
\hline & & & Double-blind Core & & \multicolumn{3}{|c|}{ FPG (mg/dL) at 26 Weeks } \\
\hline & & & $\begin{array}{c}\text { Treatment Period: } 26 \text { weeks } \\
\text { Group A: } \\
\text { placebo daily before first }\end{array}$ & & $\begin{array}{l}\text { Baseline } \\
\text { Mean } \pm \text { SD }\end{array}$ & $\begin{array}{l}\text { Adjusted } \\
\text { Mean } \\
\text { Change }\end{array}$ & $\begin{array}{l}\text { Difference } \\
\text { vs. PBO }\end{array}$ \\
\hline & & & meal of day & $\begin{array}{c}\text { PBO } \\
(\mathrm{N}=237)\end{array}$ & $156.8 \pm 38.9$ & 7.4 & - \\
\hline & & & $\begin{array}{l}\text { Group B: CANA } 100 \mathrm{mg} \\
\text { daily before first meal of }\end{array}$ & $\begin{array}{l}\text { CANA } 100 \mathrm{mg} \\
\qquad(\mathrm{N}=241)\end{array}$ & $160.4 \pm 38.7$ & -18.1 & $-25.5^{*}$ \\
\hline & & & Group C: CANA 300 mg & $\begin{array}{l}\text { CANA } 300 \mathrm{mg} \\
\quad(\mathrm{N}=236)\end{array}$ & \multirow{2}{*}{$153.2 \pm 36.6$} & -20.3 & $-27.7^{*}$ \\
\hline & & & daily before first meal of & ${ }^{*} \mathrm{p}<0.001$ vs. $\mathrm{PBC}$ & & & \\
\hline & & & & & \multicolumn{3}{|c|}{ Body Weight (kg) at 26 Weeks } \\
\hline & & & $\begin{array}{l}\text { Double-blind Extension } \\
\text { Period: } 78 \text { weeks }\end{array}$ & & $\begin{array}{l}\text { Baseline Mean } \\
\quad \pm \text { SD }\end{array}$ & $\begin{array}{c}\text { Adjusted } \\
\text { Mean } \\
\text { Percent } \\
\text { Change }\end{array}$ & $\begin{array}{l}\text { Difference } \\
\text { vs. PBO }\end{array}$ \\
\hline & & & & $\begin{array}{c}\text { PBO } \\
(\mathrm{N}=237)\end{array}$ & $91.1 \pm 17.5$ & 0.1 & - \\
\hline & & & & $\begin{array}{l}\text { CANA } 100 \mathrm{mg} \\
\qquad(\mathrm{N}=241)\end{array}$ & $88.4 \pm 15.6$ & -2.4 & $-2.3^{*}$ \\
\hline & & & & $\begin{array}{l}\text { CANA } 300 \mathrm{mg} \\
\quad(\mathrm{N}=236)\end{array}$ & $88.8 \pm 17.1$ & -3.1 & $-3.0^{*}$ \\
\hline & & & & ${ }^{*} \mathrm{p}<0.001$ vs. $\mathrm{PBC}$ & & & \\
\hline \multirow{10}{*}{$\begin{array}{l}\text { Cefalu } \\
(2013)\end{array}$} & \multirow{10}{*}{$\begin{array}{l}\text { Randomized } \\
\text { Double-blind } \\
\text { Active- } \\
\text { controlled Non- } \\
\text { inferiority }\end{array}$} & \multirow{10}{*}{$\begin{array}{c}1450 \\
\text { T2DM } \\
(756 \mathrm{M} ; \\
694 \mathrm{~F})\end{array}$} & Single-Blind Run-in Period: & & \multicolumn{3}{|c|}{ HbA1C (\%) at 52 Weeks } \\
\hline & & & $\begin{array}{l}2 \text { weeks } \\
\text { Study Phase: } 52 \text { weeks }\end{array}$ & & $\begin{array}{l}\text { Baseline } \\
\text { Mean } \pm \text { SD }\end{array}$ & $\begin{array}{l}\text { LS Mean } \\
\text { Change } \pm \text { SE }\end{array}$ & $\begin{array}{l}\text { Difference vs. } \\
\text { GLIM } \\
(95 \% \text { CI) }\end{array}$ \\
\hline & & & All groups on stable daily & $\begin{array}{c}\text { GLIM } \\
(\mathrm{N}=482)\end{array}$ & $7.8 \pm 0.8$ & $-0.81 \pm 0.04$ & - \\
\hline & & & 10 weeks plus & $\begin{array}{l}\text { CANA } 100 \mathrm{mg} \\
\qquad(\mathrm{N}=483)\end{array}$ & $7.8 \pm 0.8$ & $-0.82 \pm 0.04$ & $\begin{array}{c}-0.01 \\
(-0.11,0.09)\end{array}$ \\
\hline & & & $\begin{array}{l}\text { Group A: GLIM } 1 \text { - } 6 \mathrm{mg} \text { or } \\
1 \text { - } 8 \text { mg daily (based on }\end{array}$ & $\begin{array}{l}\text { CANA } 300 \mathrm{mg} \\
\quad(\mathrm{N}=485)\end{array}$ & $7.8 \pm 0.8$ & $-0.93 \pm 0.04$ & $\begin{array}{c}-0.12 \\
(-0.22,-0.02)\end{array}$ \\
\hline & & & country of investigational & & \multicolumn{3}{|c|}{ FPG (mg/dL) at 52 Weeks } \\
\hline & & & Group B: CANA 100 mg & & $\begin{array}{l}\text { Baseline } \\
\text { Mean } \pm \text { SD }\end{array}$ & $\begin{array}{l}\text { LS Mean } \\
\text { Change }\end{array}$ & $\begin{array}{c}\text { Difference vs. } \\
\text { GLIM } \\
(95 \% \text { CI })\end{array}$ \\
\hline & & & Group C: CANA $300 \mathrm{mg}$ & $\begin{array}{c}\text { GLIM } \\
(\mathrm{N}=482)\end{array}$ & $165.8 \pm 37.8$ & -18.4 & - \\
\hline & & & daily & $\begin{array}{l}\text { CANA } 100 \mathrm{mg} \\
\quad(\mathrm{N}=483)\end{array}$ & $165.8 \pm 37.8$ & -24.3 & $\begin{array}{c}-5.9 \\
(-10.8,-1.8)\end{array}$ \\
\hline & & & $\begin{array}{l}\text { Double-blind Extension } \\
\text { Period: } 52 \text { weeks }\end{array}$ & $\begin{array}{l}\text { CANA } 300 \mathrm{mg} \\
\qquad(\mathrm{N}=485)\end{array}$ & $164.0 \pm 36.0$ & -27.4 & $\begin{array}{c}-9.2 \\
(-12.6,-5.4)\end{array}$ \\
\hline
\end{tabular}




\section{Continued}

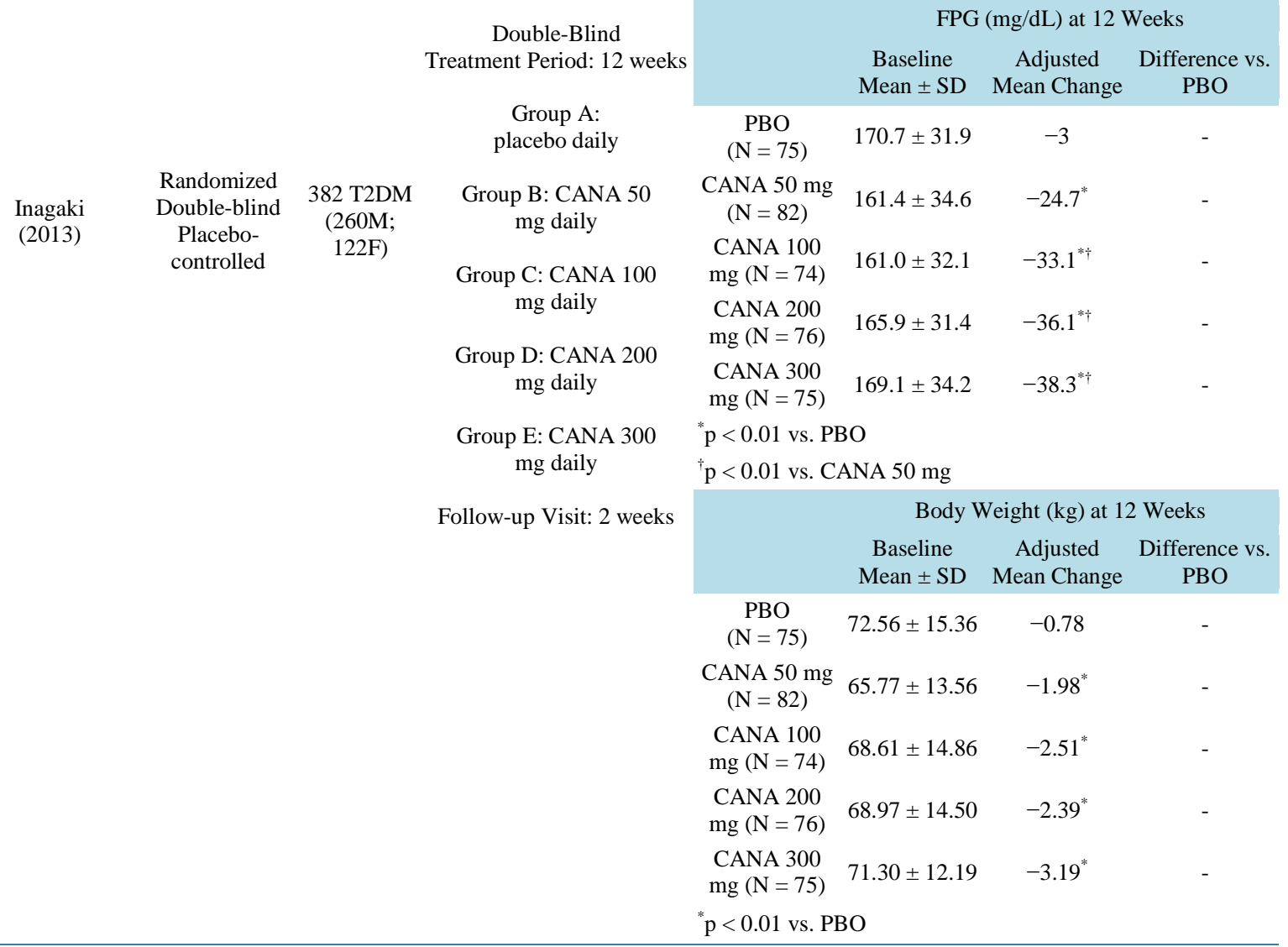

Washout Period: 8 weeks Single-blind Run-in Period: 4 weeks

Double-Blind Treatment Period: 12 weeks

Group A: placebo daily

oup B: CANA 50 C: CANA 100 D: CANA 200 E: CANA 300 daily

Body Weight (kg) at 52 Weeks

\begin{tabular}{|c|c|c|c|}
\hline & $\begin{array}{c}\text { Baseline } \\
\text { Mean } \pm \text { SD }\end{array}$ & $\begin{array}{c}\text { LS Mean } \\
\text { Percentage } \\
\text { Change } \pm \text { SE }\end{array}$ & $\begin{array}{c}\text { Difference vs. } \\
\text { GLIM } \\
(95 \% \mathrm{CI})\end{array}$ \\
\hline $\begin{array}{c}\text { GLIM } \\
(\mathrm{N}=482)\end{array}$ & $86.5 \pm 19.8$ & $1.0 \pm 0.2$ & - \\
\hline $\begin{array}{l}\text { CANA } 100 \mathrm{mg} \\
\quad(\mathrm{N}=483)\end{array}$ & $86.9 \pm 20.1$ & $-4.2 \pm 0.2$ & $\begin{array}{c}-5.2^{*} \\
(-5.7,-4.7)\end{array}$ \\
\hline $\begin{array}{l}\text { CANA } 300 \mathrm{mg} \\
\quad(\mathrm{N}=485)\end{array}$ & $86.6 \pm 19.5$ & $-4.7 \pm 0.2$ & $\begin{array}{c}-5.7^{*} \\
(-6.2,-5.1)\end{array}$ \\
\hline
\end{tabular}

${ }^{*} \mathrm{p}<0.0001$ vs. GLIM

\section{HbA1C (\%) at 12 Weeks}

$\begin{array}{cccc} & \begin{array}{c}\text { Baseline } \\ \text { Mean } \pm \text { SD }\end{array} & \begin{array}{c}\text { Adjusted } \\ \text { Mean Change }\end{array} & \begin{array}{c}\text { Difference vs. } \\ \text { PBO }\end{array} \\ \begin{array}{c}\text { PBO } \\ (\mathrm{N}=75)\end{array} & 7.99 \pm 0.77 & 0.11 & - \\ \text { CANA 50 mg } & 8.13 \pm 0.78 & -0.61^{*} & - \\ (\mathrm{N}=82) & & & \\ \text { CANA 100 } & 8.05 \pm 0.86 & -0.80^{* \dagger} & - \\ \text { mg (N = 74) } & & & \\ \text { CANA 200 } & 8.11 \pm 0.88 & -0.79^{* \dagger} & - \\ \text { mg (N = 76) } & & & - \\ \text { CANA 300 } & 8.17 \pm 0.81 & -0.88^{* \dagger} & - \\ \text { mg (N = 75) } & & & \end{array}$

$\mathrm{p}<0.01$ vs. PBO

${ }^{\dagger} \mathrm{p}<0.05$ vs. CANA $50 \mathrm{mg}$ 


\section{Continued}

$\begin{array}{ccc} & \text { Randomized } & \\ \text { Lavalle- } & \text { Double-blind } & 1284 \\ \text { González } & \text { Parallel-group } & \text { T2DM } \\ \text { (2013) } & \text { Placebo- and } & (605 \mathrm{M} ; \\ & \text { Active- } & 679 \mathrm{~F}) \\ & \text { controlled } & \end{array}$

MET and SU Dose Titration/Stabilization/ Washout Period: up to 10 weeks (if applicable)

Single-blind, Placebo Run-in Period: 2 weeks

Double-blind,

Placebo- and

Active-controlled

Treatment Period I: 26 weeks

Group A: placebo daily

Group B: SITA 100 mg daily

Group C: CANA 100 mg daily

Group D: CANA 300 mg daily

Double-blind, Active-controlled Treatment Period II: 26 weeks (Groups B-D remained the same. Group A (placebo) switched to SITA 100 mg daily)

Follow-up Period: 4 weeks

\begin{tabular}{cccc}
\multicolumn{3}{c}{ HbA1C (\%) at 26 Weeks } \\
& $\begin{array}{c}\text { Baseline } \\
\text { Mean } \pm \text { SD }\end{array}$ & $\begin{array}{c}\text { LS Mean } \\
\text { Change } \pm \text { SE }\end{array}$ & $\begin{array}{c}\text { Difference vs. } \\
\text { PBO } \\
(95 \% \text { CI })\end{array}$ \\
PBO & $8.0 \pm 0.9$ & $-0.17 \pm 0.06$ & - \\
$(\mathrm{N}=183)$ & & & \\
SITA 100 mg & $7.9 \pm 0.9$ & $-0.82 \pm 0.04$ & $-0.66^{\dagger}$ \\
$(\mathrm{N}=366)$ & & & $(-0.80,-0.52)$ \\
CANA 100 & $7.9 \pm 0.9$ & $-0.79 \pm 0.04$ & $-0.62^{*}$ \\
mg (N = 368) & & & $(-0.76,-0.48)$ \\
CANA 300 & $7.9 \pm 0.9$ & $-0.94 \pm 0.04$ & $-0.77^{*}$ \\
mg (N = 367) & & & $(-0.91,-0.64)$ \\
${ }^{*}$ p $<0.001$ vs. PBO & & &
\end{tabular}

$\mathrm{p}<0.001$ vs. $\mathrm{PBO}$

${ }^{\dagger}$ Statistical comparison vs. PBO not performed (not pre-specified)

FPG (mg/dL) at 26 Weeks

\begin{tabular}{|c|c|c|c|}
\hline & $\begin{array}{c}\text { Baseline } \\
\text { Mean } \pm \text { SD }\end{array}$ & $\begin{array}{c}\text { LS Mean } \\
\text { Change } \pm \text { SE }\end{array}$ & $\begin{array}{c}\text { Difference vs. } \\
\text { PBO } \\
(95 \% \text { CI })\end{array}$ \\
\hline $\begin{array}{c}\text { PBO } \\
(\mathrm{N}=183)\end{array}$ & $164.0 \pm 37.8$ & $1.8 \pm 1.8$ & - \\
\hline $\begin{array}{l}\text { SITA } 100 \mathrm{mg} \\
\quad(\mathrm{N}=366)\end{array}$ & $169.4 \pm 41.4$ & $-19.8 \pm 1.8$ & $\begin{array}{c}-23.4^{\dagger} \\
(-28.8,-16.2)\end{array}$ \\
\hline $\begin{array}{c}\text { CANA } 100 \\
\operatorname{mg}(N=368)\end{array}$ & $167.6 \pm 41.4$ & $-27.0 \pm 1.8$ & $\begin{array}{c}-30.6^{*} \\
(-36.0,-23.4)\end{array}$ \\
\hline $\begin{array}{c}\text { CANA } 300 \\
m g(N=367)\end{array}$ & $173.0 \pm 45.0$ & $-37.8 \pm 1.8$ & $\begin{array}{c}-39.6^{*} \\
(-46.8,-34.2)\end{array}$ \\
\hline $\mathrm{p}<0.001 \mathrm{vs}$ & & & \\
\hline
\end{tabular}

${ }^{\dagger}$ Statistical comparison vs. PBO not performed (not pre-specified)

\begin{tabular}{|c|c|c|c|}
\hline & \multicolumn{3}{|c|}{ Body Weight (kg) at 26 Weeks } \\
\hline & $\begin{array}{l}\text { Baseline } \\
\text { Mean } \pm \text { SD }\end{array}$ & $\begin{array}{c}\text { LS Mean } \\
\text { Percent } \\
\text { Change } \pm \text { SE }\end{array}$ & $\begin{array}{c}\text { Difference vs. } \\
\text { PBO } \\
(95 \% \text { CI })\end{array}$ \\
\hline $\begin{array}{c}\mathrm{PBO} \\
(\mathrm{N}=183)\end{array}$ & $86.6 \pm 22.4$ & $-1.2 \pm 0.3$ & - \\
\hline $\begin{array}{l}\text { SITA } 100 \mathrm{mg} \\
\quad(\mathrm{N}=366)\end{array}$ & $87.7 \pm 21.6$ & $-1.2 \pm 0.2$ & $\begin{array}{c}0.0^{\dagger} \\
(-0.6,0.6)\end{array}$ \\
\hline $\begin{array}{l}\text { CANA } 100 \\
m g(N=368)\end{array}$ & $88.8 \pm 22.2$ & $-3.7 \pm 0.2$ & $\begin{array}{c}-2.5^{*} \\
(-3.1,-1.9)\end{array}$ \\
\hline $\begin{array}{l}\text { CANA } 300 \\
\operatorname{mg}(N=367)\end{array}$ & $85.4 \pm 20.9$ & $-4.2 \pm 0.2$ & $\begin{array}{c}-2.9^{*} \\
(-3.5,-2.3)\end{array}$ \\
\hline \multicolumn{4}{|c|}{${ }^{*} \mathrm{p}<0.001$ vs. $\mathrm{PBO}$} \\
\hline \multicolumn{4}{|c|}{$\begin{array}{l}` \text { `Statistical comparison vs. PBO not performed (not } \\
\text { pre-specified) }\end{array}$} \\
\hline & \multicolumn{3}{|c|}{ HbA1C (\%) at 52 Weeks } \\
\hline & $\begin{array}{l}\text { Baseline } \\
\text { Mean } \pm \text { SD }\end{array}$ & $\begin{array}{l}\text { LS Mean } \\
\text { Change } \pm \text { SE }\end{array}$ & $\begin{array}{c}\text { Difference vs. } \\
\text { SITA } \\
(95 \% \text { CI })\end{array}$ \\
\hline $\begin{array}{l}\text { SITA } 100 \mathrm{mg} \\
\quad(\mathrm{N}=366)\end{array}$ & $7.9 \pm 0.9$ & $-0.73 \pm 0.05$ & - \\
\hline $\begin{array}{l}\text { CANA } 100 \\
m g(N=368)\end{array}$ & $7.9 \pm 0.9$ & $-0.73 \pm 0.05$ & $\begin{array}{c}0.00 \\
(-0.12,0.12)\end{array}$ \\
\hline
\end{tabular}




\section{Continued}

\begin{tabular}{|c|c|c|c|c|c|c|c|}
\hline & & & & $\begin{array}{c}\text { CANA } 300 \\
\mathrm{mg}(\mathrm{N}=367)\end{array}$ & $7.9 \pm 0.9$ & $-0.88 \pm 0.05$ & $\begin{array}{c}-0.15 \\
(-0.27,-0.03)\end{array}$ \\
\hline & & & & & \multicolumn{3}{|c|}{ FPG (mg/dL) at 52 Weeks } \\
\hline & & & & & $\begin{array}{c}\text { Baseline } \\
\text { Mean } \pm \text { SD }\end{array}$ & $\begin{array}{c}\text { LS Mean } \\
\text { Change } \pm \text { SE }\end{array}$ & $\begin{array}{c}\text { Difference vs. } \\
\text { SITA } \\
(95 \% \text { CI })\end{array}$ \\
\hline & & & & $\begin{array}{l}\text { SITA } 100 \mathrm{mg} \\
\quad(\mathrm{N}=366)\end{array}$ & $167.6 \pm 41.4$ & $-18.0 \pm 1.8$ & - \\
\hline & & & & $\begin{array}{l}\text { CANA } 100 \\
m g(N=368)\end{array}$ & $169.4 \pm 41.4$ & $-27.0 \pm 1.8$ & $\begin{array}{c}-9.0^{*} \\
(-12.6,-3.6)\end{array}$ \\
\hline & & & & $\begin{array}{c}\text { CANA } 300 \\
m g(N=367)\end{array}$ & $173.0 \pm 45.0$ & $-36.0 \pm 1.8$ & $\begin{array}{c}-18.0^{*} \\
(-21.6,-12.6)\end{array}$ \\
\hline & & & & \multicolumn{4}{|c|}{${ }^{*} \mathrm{p}<0.001$ vs. SITA } \\
\hline & & & & & \multicolumn{3}{|c|}{ Body Weight (kg) at 52 Weeks } \\
\hline & & & & & $\begin{array}{c}\text { Baseline } \\
\text { Mean } \pm \text { SD }\end{array}$ & $\begin{array}{c}\text { LS Mean } \\
\text { Percent } \\
\text { Change } \pm \text { SE }\end{array}$ & $\begin{array}{c}\text { Difference vs. } \\
\text { SITA } \\
\text { (95\% CI) }\end{array}$ \\
\hline & & & & $\begin{array}{l}\text { SITA } 100 \mathrm{mg} \\
\quad(\mathrm{N}=355)\end{array}$ & $87.7 \pm 21.6$ & $-1.3 \pm 0.2$ & - \\
\hline & & & & $\begin{array}{c}\text { CANA } 100 \\
m g(N=365)\end{array}$ & $88.8 \pm 22.2$ & $-3.8 \pm 0.2$ & $\begin{array}{c}-2.4^{*} \\
(-3.0,-1.8)\end{array}$ \\
\hline & & & & $\begin{array}{c}\text { CANA } 300 \\
m g(N=360)\end{array}$ & $85.4 \pm 20.9$ & $-4.2 \pm 0.2$ & $\begin{array}{c}-2.9^{*} \\
(-3.4,-2.3)\end{array}$ \\
\hline & & & & \multicolumn{4}{|c|}{${ }^{*} \mathrm{p}<0.001$ vs. SITA } \\
\hline \multirow{13}{*}{$\begin{array}{l}\text { Schernthaner } \\
\text { (2013) }\end{array}$} & \multirow{13}{*}{$\begin{array}{l}\text { Randomized } \\
\text { Double-blind } \\
\text { Active- } \\
\text { controlled }\end{array}$} & \multirow{13}{*}{$\begin{array}{l}755 \text { T2DM } \\
\text { (422M; } \\
\text { 333F) }\end{array}$} & & & \multicolumn{3}{|c|}{ HbA1C (\%) at 52 Weeks } \\
\hline & & & & & $\begin{array}{c}\text { Baseline } \\
\text { Mean } \pm \text { SD }\end{array}$ & $\begin{array}{l}\text { LS Mean } \\
\text { Change }\end{array}$ & $\begin{array}{c}\text { Difference vs. } \\
\text { SITA } \\
(95 \% \mathrm{CI})\end{array}$ \\
\hline & & & & $\begin{array}{l}\text { SITA } 100 \mathrm{mg} \\
\quad(\mathrm{N}=378)\end{array}$ & $8.1 \pm 0.9$ & -0.66 & - \\
\hline & & & $\begin{array}{l}\text { MET and SU Adjustment } \\
\text { Period (if applicable): up to } \\
12 \text { weeks } \\
\text { (Including an 8-week } \\
\text { dose-stable period) }\end{array}$ & $\begin{array}{c}\text { CANA } 300 \\
\mathrm{mg}(\mathrm{N}=377)\end{array}$ & $\begin{array}{r}8.1 \pm 0.9 \\
\text { FPG }\end{array}$ & $\begin{array}{c}-1.03 \\
(\mathrm{mg} / \mathrm{dL}) \text { at } 52 \mathrm{~V}\end{array}$ & $\begin{array}{l}\quad-0.37 \\
(-0.50,-0.25) \\
\text { Weeks }\end{array}$ \\
\hline & & & Single-blind, Placebo & & $\begin{array}{l}\text { Baseline } \\
\text { Mean }\end{array}$ & $\begin{array}{l}\text { LS Mean } \\
\text { Change }\end{array}$ & $\begin{array}{c}\text { Difference vs. } \\
\text { SITA }\end{array}$ \\
\hline & & & $\begin{array}{c}\text { Run-in Period: } 2 \text { weeks } \\
\text { Double-blind }\end{array}$ & $\begin{array}{l}\text { SITA } 100 \mathrm{mg} \\
\quad(\mathrm{N}=378)\end{array}$ & $165.8 \pm 44.9$ & -5.9 & - \\
\hline & & & Treatment Period: 52 weeks & $\begin{array}{c}\text { CANA } 300 \\
m g(N=377)\end{array}$ & $169.4 \pm 42.4$ & -29.9 & $-24.1^{*}$ \\
\hline & & & daily & ${ }^{*} \mathrm{p}<0.001$ vs. $\mathrm{S}$ & ITA & & \\
\hline & & & Group B: CANA 300 mg & & \multicolumn{3}{|c|}{ Body Weight (kg) at 52 Weeks } \\
\hline & & & $\begin{array}{c}\text { daily } \\
\text { Follow-up Period: } 4 \text { weeks }\end{array}$ & & $\begin{array}{l}\text { Baseline } \\
\text { Mean }\end{array}$ & $\begin{array}{l}\text { LS Mean } \\
\text { Percent } \\
\text { Change }\end{array}$ & $\begin{array}{c}\text { Difference vs. } \\
\text { SITA } \\
(95 \% \mathrm{CI})\end{array}$ \\
\hline & & & & $\begin{array}{c}\text { SITA } \\
(\mathrm{N}=378)\end{array}$ & $89.1 \pm 23.2$ & 0.3 & - \\
\hline & & & & $\begin{array}{l}\text { CANA } 300 \\
m g(N=377)\end{array}$ & $87.4 \pm 23.2$ & -2.5 & $-2.8^{*}$ \\
\hline & & & & \multicolumn{4}{|c|}{${ }^{*} \mathrm{p}<0.001$ vs. SITA } \\
\hline
\end{tabular}




\section{Continued}

\begin{tabular}{|c|c|c|c|c|c|c|c|}
\hline \multirow{18}{*}{$\begin{array}{l}\text { Stenlöf } \\
\text { (2013) }\end{array}$} & \multirow{18}{*}{$\begin{array}{c}\text { Randomized } \\
\text { Double-blind } \\
\text { Placebo- } \\
\text { controlled }\end{array}$} & \multirow{18}{*}{$\begin{array}{l}584 \text { T2DM } \\
\text { (258M; } \\
\text { 326F) }\end{array}$} & & & \multicolumn{3}{|c|}{ HbA1C (\%) at 26 Weeks } \\
\hline & & & & & $\begin{array}{l}\text { Baseline } \\
\text { Mean } \pm \text { SD }\end{array}$ & $\begin{array}{c}\text { LS Mean } \\
\text { Percent } \\
\text { Change }\end{array}$ & $\begin{array}{c}\text { Difference vs. } \\
\text { PBO } \\
(95 \% \mathrm{CI})\end{array}$ \\
\hline & & & & $\begin{array}{c}\text { PBO } \\
(\mathrm{N}=192)\end{array}$ & $8.0 \pm 1.0$ & 0.14 & - \\
\hline & & & & $\begin{array}{l}\text { CANA } 100 \\
\mathrm{mg}(\mathrm{N}=195)\end{array}$ & $8.1 \pm 1.0$ & -0.77 & $\begin{array}{c}-0.91^{*} \\
(-1.1,-0.7)\end{array}$ \\
\hline & & & $\begin{array}{l}\text { AHA Washout/Diet and } \\
\text { Exercise Period: } 8 \text { weeks }\end{array}$ & $\begin{array}{l}\text { CANA } 300 \\
m g(N=197)\end{array}$ & $8.0 \pm 1.0$ & -1.03 & $\begin{array}{c}-1.16^{*} \\
(-1.3,-1.0)\end{array}$ \\
\hline & & & Single-blind Placebo & ${ }^{*} \mathrm{p}<0.001$ vs. $\mathrm{P}$ & BO & & \\
\hline & & & Run-in period: 2 weeks & & FPG & $\mathrm{ng} / \mathrm{dL})$ at 26 & Neeks \\
\hline & & & $\begin{array}{c}\text { Double-blind, } \\
\text { Placebo-controlled } \\
\text { Treatment Period: } 26 \text { weeks }\end{array}$ & & $\begin{array}{l}\text { Baseline } \\
\text { Mean } \pm \text { SD }\end{array}$ & $\begin{array}{l}\text { LS Mean } \\
\text { Change }\end{array}$ & $\begin{array}{c}\text { Difference vs. } \\
\text { PBO } \\
(95 \% \text { CI })\end{array}$ \\
\hline & & & Group A: & $\begin{array}{c}\text { PBO } \\
(\mathrm{N}=192)\end{array}$ & $167.6 \pm 37.8$ & 9 & - \\
\hline & & & placebo daily & $\begin{array}{c}\text { CANA } 100 \\
m g(N=195)\end{array}$ & $173.0 \pm 43.2$ & -27 & $\begin{array}{c}-36.0^{*} \\
(-41.4,-28.8)\end{array}$ \\
\hline & & & CANA 100 mg daily & $\begin{array}{l}\text { CANA } 300 \\
m g(N=197)\end{array}$ & $173.0 \pm 43.2$ & -34.2 & $\begin{array}{c}-43.2^{*} \\
(-50.5,-36.0)\end{array}$ \\
\hline & & & $\begin{array}{l}\text { Group C: } \\
\text { CANA } 300 \text { mo dailv }\end{array}$ & ${ }^{*} \mathrm{p}<0.001$ vs. $\mathrm{P}$ & $\mathrm{BO}$ & & \\
\hline & & & 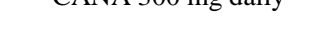 & & Body V & ight $(\mathrm{kg})$ at & 6 Weeks \\
\hline & & & $\begin{array}{c}\text { Double-blind } \\
\text { Extension Period: } 26 \text { weeks }\end{array}$ & & $\begin{array}{l}\text { Baseline } \\
\text { Mean } \pm \text { SD }\end{array}$ & $\begin{array}{c}\text { LS Mean } \\
\text { Percent } \\
\text { Change }\end{array}$ & $\begin{array}{c}\text { Difference vs. } \\
\text { PBO } \\
(95 \% \mathrm{CI})\end{array}$ \\
\hline & & & & $\begin{array}{c}\text { PBO } \\
(\mathrm{N}=192)\end{array}$ & $87.6 \pm 19.5$ & -0.6 & - \\
\hline & & & & $\begin{array}{l}\text { CANA } 100 \\
m g(N=195)\end{array}$ & $85.8 \pm 21.4$ & -2.8 & $\begin{array}{c}-2.2^{*} \\
(-2.9,-1.6)\end{array}$ \\
\hline & & & & $\begin{array}{l}\text { CANA } 300 \\
\mathrm{mg}(\mathrm{N}=197)\end{array}$ & $86.9 \pm 20.5$ & -3.9 & $\begin{array}{c}-3.3^{*} \\
(-4.0,-2.6)\end{array}$ \\
\hline & & & & ${ }^{*} \mathrm{p}<0.001$ vs. $\mathrm{P}$ & $\mathrm{BO}$ & & \\
\hline \multirow{11}{*}{$\begin{array}{l}\text { Wilding } \\
\text { (2013) }\end{array}$} & \multirow{11}{*}{$\begin{array}{c}\text { Randomized } \\
\text { Double-blind } \\
\text { Placebo- } \\
\text { controlled }\end{array}$} & \multirow{11}{*}{$\begin{array}{l}469 \text { T2DM } \\
\text { (239M; } \\
\text { 230F) }\end{array}$} & \multirow{3}{*}{$\begin{array}{l}\text { MET and SU Maximum } \\
\text { Effective Dose Pretreatment } \\
\text { Period: up to } 12 \text { weeks } \\
\text { (dose titration up to } 4 \\
\text { weeks; stable dose for } 8 \\
\text { weeks) }\end{array}$} & & \multicolumn{3}{|c|}{ HbA1C (\%) at 26 Weeks } \\
\hline & & & & & $\begin{array}{l}\text { Baseline } \\
\text { Mean } \pm \text { SD }\end{array}$ & $\begin{array}{l}\text { LS Mean } \\
\text { Change }\end{array}$ & $\begin{array}{c}\text { Difference vs. } \\
\text { PBO } \\
(95 \% \mathrm{CI})\end{array}$ \\
\hline & & & & $\begin{array}{c}\text { PBO } \\
(\mathrm{N}=156)\end{array}$ & $8.1 \pm 0.9$ & -0.13 & - \\
\hline & & & $\begin{array}{l}\text { Single-blind, Placebo } \\
\text { Run-in Period: } 2 \text { weeks }\end{array}$ & $\begin{array}{l}\text { CANA } 100 \\
\mathrm{mg}(\mathrm{N}=157)\end{array}$ & $8.1 \pm 0.9$ & -0.85 & $\begin{array}{c}-0.71^{*} \\
(-0.90,-0.52)\end{array}$ \\
\hline & & & \multirow{2}{*}{$\begin{array}{l}\text { Double-blind Treatment } \\
\text { Period: } 26 \text { weeks }\end{array}$} & $\begin{array}{c}\text { CANA } 300 \\
m g(N=156)\end{array}$ & $8.1 \pm 0.9$ & -1.06 & $\begin{array}{c}-0.92^{*} \\
(-1.11,-0.73)\end{array}$ \\
\hline & & & & \multicolumn{3}{|c|}{${ }^{*} \mathrm{p}<0.001$ vs. $\mathrm{PBO}$} & \\
\hline & & & Group A: placebo daily & & \multicolumn{3}{|c|}{ FPG (mg/dL) at 26 Weeks } \\
\hline & & & $\begin{array}{l}\text { Group B: CANA } 100 \mathrm{mg} \\
\text { daily before first meal }\end{array}$ & & $\begin{array}{c}\text { Baseline } \\
\text { Mean } \pm \text { SD }\end{array}$ & $\begin{array}{l}\text { LS Mean } \\
\text { Change }\end{array}$ & $\begin{array}{c}\text { Difference vs. } \\
\text { PBO } \\
(95 \% \mathrm{CI})\end{array}$ \\
\hline & & & \multirow{2}{*}{$\begin{array}{l}\text { Group C: CANA } 300 \mathrm{mg} \\
\text { daily before first meal }\end{array}$} & $\begin{array}{c}\text { PBO } \\
(\mathrm{N}=156)\end{array}$ & $169.4 \pm 39.6$ & 3.6 & - \\
\hline & & & & $\begin{array}{l}\text { CANA } 100 \\
\mathrm{mg}(\mathrm{N}=157)\end{array}$ & $173.0 \pm 41.4$ & -18 & $\begin{array}{c}-21.6^{*} \\
(-30.6,-12.6)\end{array}$ \\
\hline & & & $\begin{array}{l}\text { Double-blind Extension } \\
\text { Period: } 26 \text { weeks }\end{array}$ & $\begin{array}{l}\text { CANA } 300 \\
\mathrm{mg}(\mathrm{N}=156)\end{array}$ & $167.6 \pm 37.8$ & -30.6 & $\begin{array}{c}-34.2^{*} \\
(-43.2,-25.2)\end{array}$ \\
\hline
\end{tabular}




\section{Continued}

${ }^{*} \mathrm{p}<0.001$ vs. $\mathrm{PBO}$

\begin{tabular}{|c|c|c|c|}
\hline & \multicolumn{3}{|c|}{ Body Weight (kg) at 26 Weeks } \\
\hline & $\begin{array}{c}\text { Baseline } \\
\text { Mean } \pm \text { SD }\end{array}$ & $\begin{array}{l}\text { LS Mean } \\
\text { Percent } \\
\text { Change }\end{array}$ & $\begin{array}{c}\text { Difference vs. } \\
\text { PBO } \\
(95 \% \text { CI })\end{array}$ \\
\hline $\begin{array}{c}\text { PBO } \\
(\mathrm{N}=156)\end{array}$ & $91.2 \pm 22.6$ & -0.7 & - \\
\hline $\begin{array}{c}\text { CANA } 100 \\
\mathrm{mg}(\mathrm{N}=157)\end{array}$ & $93.8 \pm 22.6$ & -2.1 & $\begin{array}{c}-1.4^{*} \\
(-2.1,-0.7)\end{array}$ \\
\hline $\begin{array}{c}\text { CANA } 300 \\
\mathrm{mg}(\mathrm{N}=156)\end{array}$ & $93.5 \pm 22.0$ & -2.6 & $\begin{array}{c}-2.0^{*} \\
(-2.7,-1.3)\end{array}$ \\
\hline
\end{tabular}

\begin{tabular}{cccc} 
& \multicolumn{3}{c}{ HbA1C (\%) at 52 Weeks } \\
& $\begin{array}{c}\text { Baseline } \\
\text { Mean } \pm \text { SD }\end{array}$ & $\begin{array}{c}\text { LS Mean } \\
\text { Change }\end{array}$ & $\begin{array}{c}\text { Difference vs. } \\
\text { PBO } \\
(95 \% \text { CI })\end{array}$ \\
PBO & $8.1 \pm 0.9$ & 0.01 & - \\
$(\mathrm{N}=156)$ & & & -0.75 \\
CANA 100 mg & $8.1 \pm 0.9$ & -0.74 & $(-0.95,-0.55)$ \\
$(\mathrm{N}=157)$ & & & -0.97 \\
CANA 300 mg \\
$(\mathrm{N}=156)$
\end{tabular}

\begin{tabular}{|c|c|c|c|}
\hline & \multicolumn{3}{|c|}{ FPG (mg/dL) at 52 Weeks } \\
\hline & $\begin{array}{c}\text { Baseline } \\
\text { Mean } \pm \text { SD }\end{array}$ & $\begin{array}{l}\text { LS Mean } \\
\text { Change }\end{array}$ & $\begin{array}{c}\text { Difference vs. } \\
\text { PBO } \\
(95 \% \mathrm{CI})\end{array}$ \\
\hline $\begin{array}{c}\text { PBO } \\
(\mathrm{N}=156)\end{array}$ & $169.4 \pm 39.6$ & 10.8 & - \\
\hline $\begin{array}{l}\text { CANA } 100 \mathrm{mg} \\
\quad(\mathrm{N}=157)\end{array}$ & $173.0 \pm 41.4$ & -19.8 & $\begin{array}{c}-28.8 \\
(-37.8,-19.8)\end{array}$ \\
\hline $\begin{array}{l}\text { CANA } 300 \mathrm{mg} \\
\quad(\mathrm{N}=156)\end{array}$ & $167.6 \pm 37.8$ & -27 & $\begin{array}{c}-37.8 \\
(-46.8,-28.8)\end{array}$ \\
\hline
\end{tabular}

\begin{tabular}{|c|c|c|c|}
\hline & \multicolumn{3}{|c|}{ Body Weight (kg) at 52 Weeks } \\
\hline & $\begin{array}{c}\text { Baseline } \\
\text { Mean } \pm \text { SD }\end{array}$ & $\begin{array}{l}\text { LS Mean } \\
\text { Percent } \\
\text { Change }\end{array}$ & $\begin{array}{c}\text { Difference vs. } \\
\text { PBO } \\
(95 \% \mathrm{CI})\end{array}$ \\
\hline $\begin{array}{c}\text { PBO } \\
(\mathrm{N}=156)\end{array}$ & $91.2 \pm 22.6$ & -0.9 & - \\
\hline $\begin{array}{l}\text { CANA } 100 \mathrm{mg} \\
\quad(\mathrm{N}=157)\end{array}$ & $93.8 \pm 22.6$ & -2.2 & $\begin{array}{c}-1.3 \\
(-2.1,-0.5)\end{array}$ \\
\hline $\begin{array}{l}\text { CANA } 300 \mathrm{mg} \\
\quad(\mathrm{N}=156)\end{array}$ & $93.5 \pm 22.0$ & -3.2 & $\begin{array}{c}-2.2 \\
(-3.0,-1.4)\end{array}$ \\
\hline
\end{tabular}
Yale Double-blind (2013) controlled

269 T2DM
with
chronic
kidney
disease
(163M;
106F)

AHA Dose Titration Period (if required): up to 4 weeks

AHA Dose Stable Period (if required): 8 weeks

Single-blind, Placebo Run-in Period: 2 weeks

Double-blind Core Treatment Period: 26 weeks

\begin{tabular}{lccc} 
& \multicolumn{3}{c}{ HbA1C (\%) at 26 Weeks } \\
& $\begin{array}{c}\text { Baseline } \\
\text { Mean } \pm \text { SD }\end{array}$ & $\begin{array}{c}\text { LS Mean } \\
\text { Change }\end{array}$ & $\begin{array}{c}\text { Difference vs. } \\
\text { PBO } \\
(95 \% \mathrm{CI})\end{array}$ \\
PBO & $8.0 \pm 0.9$ & -0.03 & - \\
$(\mathrm{N}=90)$ & & & $-0.30^{*}$ \\
CANA 100 mg & $7.9 \pm 0.9$ & -0.33 & $(-0.5,-0.1)$ \\
$(\mathrm{N}=90)$ & & & $-0.40^{* *}$ \\
CANA 300 mg & $8.0 \pm 0.8$ & -0.44 & $(-0.6,-0.2)$ \\
$\quad(\mathrm{N}=89)$ & & & \\
$\mathrm{p}<0.05$ vs. PBO & & & \\
${ }^{* * *} \mathrm{p}<0.001$ vs. PBO & & \\
\hline
\end{tabular}




\section{Continued}

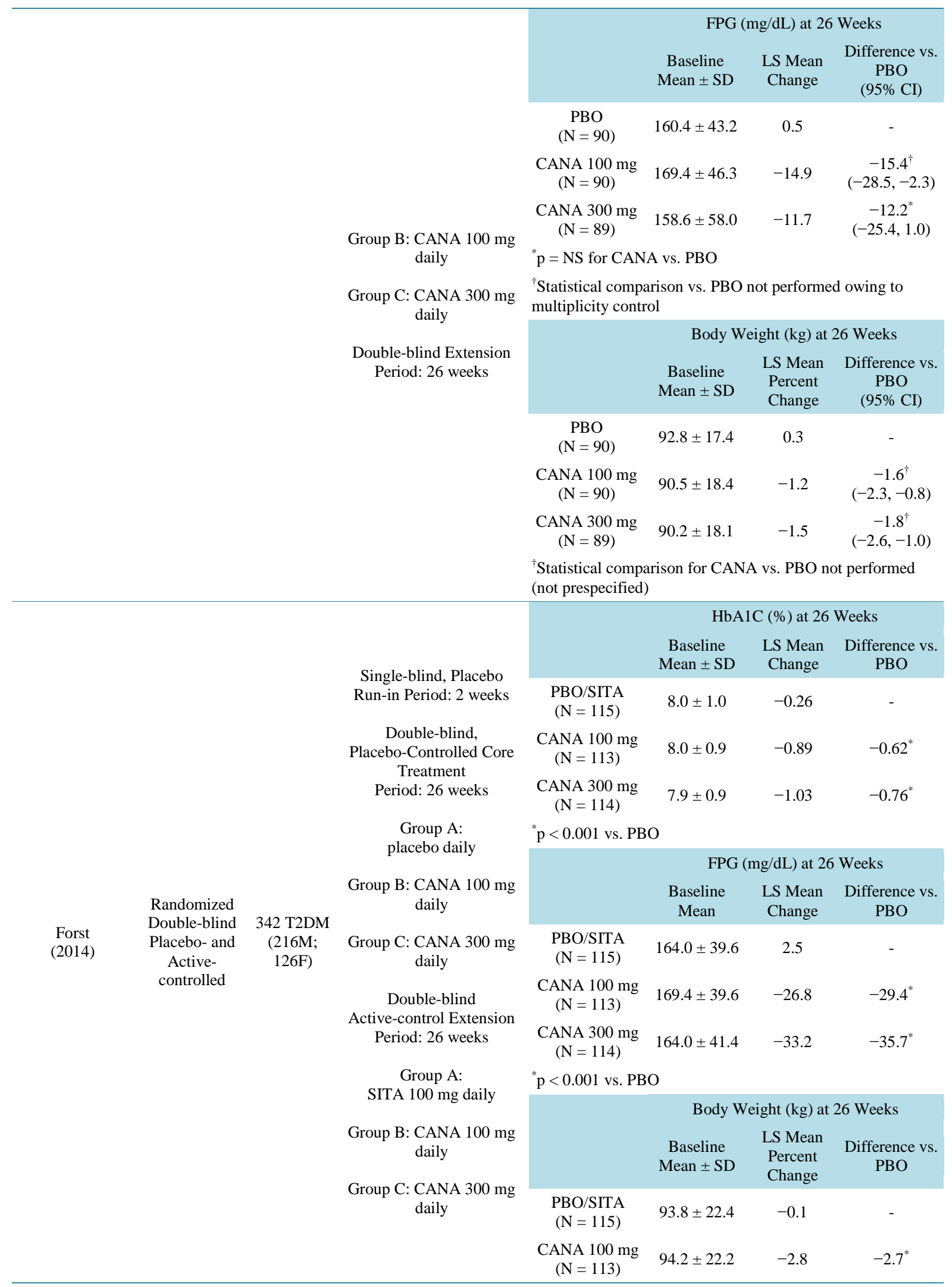




\section{Continued}

\begin{tabular}{|c|c|c|c|}
\hline $\begin{array}{l}\text { CANA } 300 \mathrm{mg} \\
\quad(\mathrm{N}=114)\end{array}$ & $94.4 \pm 25.9$ & -3.8 & $-3.7^{*}$ \\
\hline \multicolumn{4}{|c|}{ * $\mathrm{p}<0.001$ vs. $\mathrm{PBO}$} \\
\hline & \multicolumn{3}{|c|}{ HbA1C (\%) at 52 Weeks } \\
\hline & $\begin{array}{c}\text { Baseline } \\
\text { Mean } \pm \text { SD }\end{array}$ & $\begin{array}{l}\text { LS Mean } \\
\text { Change } \\
(95 \% \text { CI })\end{array}$ & $\begin{array}{l}\text { LS Mean } \\
\text { Change } \\
(95 \% \text { CI })\end{array}$ \\
\hline $\begin{array}{c}\mathrm{PBO} / \mathrm{SITA} \\
(\mathrm{N}=115)\end{array}$ & $8.0 \pm 1.0$ & - & - \\
\hline $\begin{array}{l}\text { CANA } 100 \mathrm{mg} \\
\quad(\mathrm{N}=113)\end{array}$ & $8.0 \pm 0.9$ & $\begin{array}{c}-0.92 \\
(-1.06,-0.79)\end{array}$ & - \\
\hline \multirow[t]{3}{*}{$\begin{array}{l}\text { CANA } 300 \mathrm{mg} \\
\quad(\mathrm{N}=114)\end{array}$} & $7.9 \pm 0.9$ & $\begin{array}{c}-1.03 \\
(-1.17,-0.89)\end{array}$ & - \\
\hline & \multicolumn{3}{|c|}{ FPG (mg/dL) at 52 Weeks } \\
\hline & $\begin{array}{l}\text { Baseline } \\
\text { Mean }\end{array}$ & $\begin{array}{l}\text { LS Mean } \\
\text { Change } \\
(95 \% \text { CI })\end{array}$ & $\begin{array}{c}\text { Difference } \\
\text { vs. PBO }\end{array}$ \\
\hline $\begin{array}{c}\mathrm{PBO} / \mathrm{SITA} \\
(\mathrm{N}=115)\end{array}$ & $164.0 \pm 39.6$ & - & - \\
\hline $\begin{array}{l}\text { CANA } 100 \mathrm{mg} \\
\quad(\mathrm{N}=113)\end{array}$ & $169.4 \pm 39.6$ & $\begin{array}{c}-26.7 \\
(-32.4,-21.1)\end{array}$ & - \\
\hline \multirow[t]{3}{*}{$\begin{array}{l}\text { CANA } 300 \mathrm{mg} \\
\quad(\mathrm{N}=114)\end{array}$} & $164.0 \pm 41.4$ & $\begin{array}{c}-31.5 \\
(-37.2,-25.8)\end{array}$ & - \\
\hline & \multicolumn{3}{|c|}{ Body Weight (kg) at 52 Weeks } \\
\hline & $\begin{array}{l}\text { Baseline } \\
\text { Mean }\end{array}$ & $\begin{array}{c}\text { LS Mean } \\
\text { Percent } \\
\text { Change } \\
\text { (95\% CI) }\end{array}$ & $\begin{array}{l}\text { Difference } \\
\text { vs. PBO }\end{array}$ \\
\hline $\begin{array}{c}\mathrm{PBO} / \mathrm{SITA} \\
(\mathrm{N}=115)\end{array}$ & $93.8 \pm 22.4$ & - & - \\
\hline $\begin{array}{l}\text { CANA } 100 \mathrm{mg} \\
\quad(\mathrm{N}=113)\end{array}$ & $94.2 \pm 22.2$ & $\begin{array}{c}-2.7 \\
(-3.6,-1.9)\end{array}$ & - \\
\hline $\begin{array}{l}\text { CANA } 300 \mathrm{mg} \\
\quad(\mathrm{N}=114)\end{array}$ & $94.4 \pm 25.9$ & $\begin{array}{c}-3.7 \\
(-4.6,-2.9)\end{array}$ & - \\
\hline
\end{tabular}

95\% CI = 95\% confidence interval; AHA = antihyperglycemic agent; $\mathrm{AM}=$ morning; $\mathrm{CANA}=$ canagliflozin; $\mathrm{F}=$ Female; FPG = Fasting Plasma Glucose; GLIM = glimepiride; HbA1C = hemoglobin A1C; LS = least squares; $\mathrm{M}=$ Male; MET = metformin; NS = not significant; PBO = placebo; $\mathrm{SD}$ = standard deviation; $\mathrm{SE}$ = standard error; SITA = sitagliptin; $\mathrm{SU}=$ sulfonylurea; T2DM = Type 2 Diabetes Mellitus Patients

liflozin is 91\% protein bound, which is unchanged in patients with renal or hepatic impairment. It is metabolized primarily by UGT1A9 with minor CYP-activity and is eliminated mainly through the kidneys. At steady state, T2DM patients with mild, moderate, or severe renal impairment (determined by estimated glomerular filtration rate (eGFR)) experience $45 \%$ to 3 -fold higher systemic exposure of the drug without a corresponding higher 24-hour urinary glucose excretion. The steady state 24 -hour urinary glucose excretion is $42 \%-90 \%$ lower in these patients [2].

Dapagliflozin is not recommended in patients with moderate renal impairment as improvement in glycemic control was not seen in this population. Additionally, dapagliflozin is not expected to be effective in patients with severe renal impairment or end stage renal disease (ESRD). Dapagliflozin is contraindicated in these populations along with patients on dialysis. According to Child-Pugh class grading, mild, moderate, and severe hepatic impairment do not warrant dose adjustments with dapagliflozin, but the risk-benefit for use in patients with severe impairment should be individually assessed as the safety and efficacy have not been specifically studied in this population [2].

Overall, 11 studies assessing the use of dapagliflozin in T2DM were identified (Table 2) [18]-[28]. The majority of trials evaluated the effectiveness of dapagliflozin as add-on therapy to standard treatments [18] [20] [22] [24]-[28]. The time period for the 11 studies ranged from 12 to 102 weeks, and investigators used dapagliflozin 
doses of 1 - $20 \mathrm{mg}$ [18]-[28]. Dapagliflozin consistently showed statistically significant decreases in mean HbA1C (\%) compared to control groups [18]-[23] [25] [26] [28]. The largest difference in HbA1C (\%) reduction between dapagliflozin and placebo groups was $-0.84 \%$ after 24 weeks of treatment with dapagliflozin $5 \mathrm{mg}$ [19]. No difference in the adjusted mean change in HbA1C (\%) from baseline between the dapagliflozin and glipizide groups was observed in a 52-week study, which concluded that dapagliflozin was statistically noninferior to glipizide [24]. A few trials found a statistically significant difference in patients achieving an HbA1C of $<7 \%$ at the end of the study period in the dapagliflozin treatment groups compared to placebo [18]-[20] [22] [26].

The majority of studies found a statistically significant decrease with mean FPG in dapagliflozin treatment groups compared to control groups [18]-[23] [25] [26] [28]. Three of the four studies that assessed 2-h PPG after an oral glucose tolerance test (OGTT) found statistically significant decreases in dapagliflozin groups compared to control groups [19] [25] [26]. Although [27] did not report statistical significance, 2-h PPG was found to be lower in the dapagliflozin groups. All studies found a greater decrease in body weight after treatment with dapagliflozin compared to the control [18]-[28].

The most common adverse events were diarrhea, headache, nasopharyngitis, upper respiratory tract infection, urinary tract infection, influenza, back pain, hypertension, cough, and arthralgia [18]-[28]. Nine trials documented $>55 \%$ of subjects experiencing at least one adverse event but $<10 \%$ of subjects discontinued a study due to an adverse event [18]-[22] [24] [25] [27] [28]. Fewer adverse events overall were reported by [23] (38.9\% $53.8 \%$ ) and [26] (47.3\% - 51.9\%). Of the reported events, approximately $25 \%$ or fewer were determined to be drug-related in five trials [18]-[20] [22] [26]. The overall incidence of urinary and genital tract infections was low ( $<15 \%)$, but more common in dapagliflozin groups compared to control groups [18]-[25] [27] [28]. In five of the studies, the events were reported as mild or moderate and responded adequately to treatment [18]-[21] [26]. Among four other trials, there was a total of 12 patients who withdrew from the study because of a UTI or genital infection [24]-[26] [28].

Hypoglycemic events did not occur frequently $(<10 \%)$ or severely and were similar to placebo or treatment groups in most studies [18]-[23] [25]. The incidence of hypoglycemia was higher in treatment groups using dapagliflozin with hypoglycemia-associated medications such as insulin (25\% - $29.2 \%$ versus $13 \%$ in placebo) [28], insulin with insulin-sensitizers (53.6\% - 60.4\% versus 51.8\% in placebo) [27] and sulfonylureas (6.9\% $7.9 \%$ versus $4.8 \%$ in placebo) [26]. A serious adverse event related to hypoglycemia was also reported for dapagliflozin $5 \mathrm{mg}$ [28]. A significantly higher proportion of patients experienced hypoglycemia on glipizide (40.8\%) compared to dapagliflozin (3.5\%) with six episodes of hypoglycemia leading to discontinuation in the glipizide group [24].

Blood pressure was also monitored in trials. Some studies reported that systolic blood pressure was slightly reduced in both dapagliflozin and control groups [18] [21] [22] [26] [28], while others reported that it was lowered only in dapagliflozin treatment groups compared to control groups [19] [20] [23]-[25] [27]. Overall, most studies found that dapagliflozin treatment was associated with only a few incidences of hypotension $(<5 \%)$ [18]-[22] [24] [28]. An incidence of syncope was reported in a patient receiving dapagliflozin $10 \mathrm{mg}$ [26], while an episode of severe hypotension was noted in a patient on dapagliflozin $5 \mathrm{mg}$ [19].

\subsection{Empagliflozin}

Approved by the FDA in August 2014, empagliflozin, D-Glucitol,1,5-anhydro-1-C-[4-chloro-3-[[4-[[(3S)-tetrahydro-3-furanyl]oxy]phenyl]methyl]phenyl]-, (1S), is the newest SGLT2 inhibitor to enter the market. Similar to the other available agents, empagliflozin is approved for use in T2DM patients, as an adjunct to diet and exercise [3]. Plasma concentrations peak at approximately 1.5 hours post-oral administration [3] [29] with a reduction in AUC (16\%) and $\mathrm{C}_{\max }(37 \%)$ when taken after a high-fat and high-calorie meal. Although reductions in systemic exposure were noted, the impact on clinical outcomes was not deemed significant. As a result, empagliflozin may be taken with or without food. Empagliflozin has a plasma protein binding of roughly 86\%. Metabolism occurs primarily via glucuronidation with minimal metabolite exposure. The terminal half-life of empagliflozin is 12.4 hours. Empagliflozin is primarily eliminatedrenally. Increases in AUC have occurred in patients with renal impairment, kidney failure or ESRD. Empagliflozin is contraindicated in severe renal impairment, ESRD, or dialysis [3].

The impact on plasma concentration varies based on the degree of renal and hepatic impairment. Patients with moderate renal impairment, kidney failure, or ESRD have peak plasma concentrations comparable to patients 
Table 2. Dapagliflozin trials.

\begin{tabular}{|c|c|c|c|c|c|c|c|}
\hline $\begin{array}{l}\text { Author } \\
\text { (Year) }\end{array}$ & Study Design & Subjects & Methods & \multicolumn{4}{|c|}{ Results } \\
\hline \multirow{17}{*}{$\begin{array}{l}\text { Wilding } \\
\text { (2009) }\end{array}$} & \multirow{17}{*}{$\begin{array}{c}\text { Randomized } \\
\text { Double-blind } \\
\text { Placebo- } \\
\text { controlled }\end{array}$} & \multirow{17}{*}{$\begin{array}{l}71 \mathrm{~T} 2 \mathrm{DM} \\
(42 \mathrm{M} ; 29 \mathrm{~F})\end{array}$} & \multirow{7}{*}{$\begin{array}{c}\text { Stabilization of } \\
\text { Insulin Sensitizer } \\
\text { Therapy and } \\
\text { Insulin: } \geq 6 \text { weeks } \\
\text { with insulin } \\
\text { treatment for } \geq 12 \\
\text { weeks }\end{array}$} & & \multicolumn{3}{|c|}{ HbA1C (\%) at 12 Weeks } \\
\hline & & & & & Baseline Mean \pm SD & $\begin{array}{l}\text { Adjusted Mean } \\
\text { Change (95\% } \\
\text { CI) }\end{array}$ & $\begin{array}{l}\text { Difference vs. } \\
\text { PBO (95\% CI) }\end{array}$ \\
\hline & & & & $\mathrm{PBO}(\mathrm{N}=23)$ & $8.4 \pm 0.9$ & $\begin{array}{c}0.03 \\
(-0.2,0.4)\end{array}$ & - \\
\hline & & & & $\begin{array}{l}\text { DAPA } 10 \mathrm{mg} \\
(\mathrm{N}=24)\end{array}$ & $8.4 \pm 0.7$ & $\begin{array}{c}-0.61 \\
(-0.9,-0.4)\end{array}$ & $\begin{array}{c}-0.7 \\
(-1.1,-0.3)\end{array}$ \\
\hline & & & & $\begin{array}{l}\text { DAPA } 20 \mathrm{mg} \\
(\mathrm{N}=24)\end{array}$ & $8.5 \pm 0.9$ & $\begin{array}{c}-0.69 \\
(-0.9,-0.4)\end{array}$ & $\begin{array}{c}-0.78 \\
(-1.2,-0.4)\end{array}$ \\
\hline & & & & & FPG (n & $\mathrm{ng} / \mathrm{dL})$ at $12 \mathrm{We}$ & \\
\hline & & & & & Baseline Mean \pm SD & $\begin{array}{l}\text { Adjusted Mean } \\
\text { Change (95\% } \\
\text { CI) }\end{array}$ & $\begin{array}{l}\text { Difference vs. } \\
\text { PBO (95\% CI) }\end{array}$ \\
\hline & & & $\begin{array}{l}\text { Double-blind } \\
\text { Treatment Period: }\end{array}$ & $\mathrm{PBO}(\mathrm{N}=23)$ & $165.9 \pm 51.5$ & $\begin{array}{c}17.8 \\
(1.4,34.2)\end{array}$ & - \\
\hline & & & 12 wеeкs & DAPA $10 \mathrm{mg}$ & $1560+390$ & 2.4 & -15.4 \\
\hline & & & $\begin{array}{l}\text { Group A: } \\
\text { placebo dailv + }\end{array}$ & $(\mathrm{N}=24)$ & $156.0 \pm 39.0$ & $(-13.6,18.3)$ & $(-38.4,7.5)$ \\
\hline & & & insulin & & & -9.6 & -27.4 \\
\hline & & & $\begin{array}{l}\text { Group B: DAPA } 10 \\
\text { mg daily + insulin }\end{array}$ & $\begin{array}{l}\text { DAPA } 20 \mathrm{mg} \\
(\mathrm{N}=24)\end{array}$ & $161.6 \pm 55.0$ & $(-25.6,6.3)$ & $(-50.3,-4.6)$ \\
\hline & & & Group C: DAPA 20 & & Body We & eight $(\mathrm{kg})$ at $12 \mathrm{~V}$ & Jeeks \\
\hline & & & mg daily + insulin & & Baseline Mean \pm SD & $\begin{array}{c}\text { Adjusted Mean } \\
\text { Change (95\% } \\
\text { CI) }\end{array}$ & $\begin{array}{c}\text { Difference vs. } \\
\text { PBO } \\
(95 \% \mathrm{CI})\end{array}$ \\
\hline & & & & $\mathrm{PBO}(\mathrm{N}=23)$ & $101.8 \pm 16.5$ & $\begin{array}{c}-1.9 \\
(-2.9,-0.9)\end{array}$ & - \\
\hline & & & & $\begin{array}{l}\text { DAPA } 10 \mathrm{mg} \\
\quad(\mathrm{N}=24)\end{array}$ & $103.4 \pm 10.2$ & $\begin{array}{c}-4.5 \\
(-5.5,-3.5)\end{array}$ & $\begin{array}{c}-2.6 \\
(-4.0,-1.2)\end{array}$ \\
\hline & & & & $\begin{array}{l}\text { DAPA } 20 \mathrm{mg} \\
\quad(\mathrm{N}=24)\end{array}$ & $101.2 \pm 15.3$ & $\begin{array}{c}-4.3 \\
(-5.3,-3.3)\end{array}$ & $\begin{array}{c}-2.4 \\
(-3.8,-1.0)\end{array}$ \\
\hline \multirow{6}{*}{$\begin{array}{l}\text { Bailey } \\
(2010)\end{array}$} & \multirow{6}{*}{$\begin{array}{c}\text { Randomized } \\
\text { Double-blind } \\
\text { Parallel Group } \\
\text { Placebo- } \\
\text { Controlled }\end{array}$} & \multirow{6}{*}{$\begin{array}{c}\text { 546 T2DM } \\
\text { (292M; 254F) }\end{array}$} & $\begin{array}{l}\text { Single-blind, } \\
\text { Placebo Lead-in } \\
\text { Period : } 2 \text { weeks } \\
\text { Double-blind }\end{array}$ & & Baseline Mean \pm SD & $\begin{array}{l}\text { C (\%) at } 24 \text { Wee } \\
\text { Adjusted Mean } \\
\text { Change (95\% } \\
\text { CI) }\end{array}$ & $\begin{array}{l}\text { Difference vs. } \\
\text { PBO (95\% CI) }\end{array}$ \\
\hline & & & $\begin{array}{l}\text { Treatment Period: } \\
24 \text { weeks } \\
\text { (All groups on }\end{array}$ & $\mathrm{PBO}(\mathrm{N}=137)$ & $8.11 \pm 0.96$ & $\begin{array}{c}-0.3 \\
(-0.44,-0.16)\end{array}$ & - \\
\hline & & & $\begin{array}{l}\text { stable MET dose) } \\
\text { Group A: placebo + }\end{array}$ & $\begin{array}{l}\text { DAPA } 2.5 \mathrm{mg} \\
\quad(\mathrm{N}=137)\end{array}$ & $7.99 \pm 0.90$ & $\begin{array}{c}-0.67^{* \dagger} \\
(-0.81,-0.53)\end{array}$ & - \\
\hline & & & $\begin{array}{l}\text { MET daily in AM } \\
\text { Group B: DAPA }\end{array}$ & $\begin{array}{l}\text { DAPA } 5 \mathrm{mg} \\
(\mathrm{N}=137)\end{array}$ & $8.17 \pm 0.96$ & $\begin{array}{c}-0.70^{* * \dagger} \\
(-0.85,-0.56)\end{array}$ & - \\
\hline & & & $\begin{array}{c}2.5 \mathrm{mg}+\mathrm{MET} \\
\text { daily in AM }\end{array}$ & $\begin{array}{l}\text { DAPA } 10 \mathrm{mg} \\
(\mathrm{N}=135)\end{array}$ & $7.92 \pm 0.82$ & $\begin{array}{c}-0.84^{* * \dagger} \\
(-0.98,-0.70)\end{array}$ & - \\
\hline & & & $\begin{array}{l}\text { Group C: DAPA } 5 \\
\text { mg + MET daily in } \\
\text { AM }\end{array}$ & \multicolumn{4}{|c|}{$\begin{array}{l}{ }^{*} \mathrm{p}=0.0002 \\
{ }^{* *} \mathrm{p}<0.0001\end{array}$} \\
\hline
\end{tabular}




\section{Continued}

\begin{tabular}{|c|c|c|c|c|c|c|c|}
\hline & & & & ${ }^{\dagger}$ Significant vs. I & BO at $\alpha=0.019$ ap & lying Dunnett's ad & djustment \\
\hline & & & & & FPG & $\mathrm{ng} / \mathrm{dL}$ ) at $24 \mathrm{Week}$ & \\
\hline & & & & & $\begin{array}{l}\text { Baseline Mean } \pm \\
\text { SD }\end{array}$ & $\begin{array}{l}\text { Adjusted Mean } \\
\text { Change (95\% } \\
\text { CI) }\end{array}$ & $\begin{array}{l}\text { Difference vs. } \\
\text { PBO (95\% CI) }\end{array}$ \\
\hline & & & & $\mathrm{PBO}(\mathrm{N}=137)$ & $165.6 \pm 46.3$ & $\begin{array}{c}-5.9 \\
(-11.2,-0.7)\end{array}$ & - \\
\hline & & & & $\begin{array}{l}\text { DAPA } 2.5 \mathrm{mg} \\
(\mathrm{N}=137)\end{array}$ & $161.4 \pm 43.1$ & $\begin{array}{c}-17.8^{* \dagger} \\
(-23.1,-12.4)\end{array}$ & - \\
\hline & & & & $\begin{array}{l}\text { DAPA } 5 \mathrm{mg} \\
(\mathrm{N}=137)\end{array}$ & $169.2 \pm 49.0$ & $\begin{array}{c}-21.4^{* * \dagger} \\
(-26.8,-16.2)\end{array}$ & - \\
\hline & & & $\begin{array}{c}\text { Group D: DAPA } 10 \\
\text { mg + MET daily in } \\
\text { AM }\end{array}$ & $\begin{array}{l}\text { DAPA } 10 \mathrm{mg} \\
\quad(\mathrm{N}=135) \\
{ }^{*} \mathrm{p}=0.0019 \\
{ }^{* *} \mathrm{p}<0.0001\end{array}$ & $156.0 \pm 38.7$ & $\begin{array}{c}-23.4^{* * \dagger} \\
(-28.8,-18.0)\end{array}$ & - \\
\hline & & & Double-blind & ${ }^{\dagger}$ Significant after & sequential testing $\mathrm{p}$ & ocedure at $\alpha=0.05$ & \\
\hline & & & to 102 weeks (refer & & Body W & eight $(\mathrm{kg})$ at $24 \mathrm{We}$ & eeks \\
\hline & & & to Bailey 2013) & & $\begin{array}{c}\text { Baseline Mean } \pm \\
\text { SD }\end{array}$ & $\begin{array}{l}\text { Adjusted Mean } \\
\text { Change (95\% } \\
\text { CI) }\end{array}$ & $\begin{array}{c}\text { Difference vs. } \\
\text { PBO }\end{array}$ \\
\hline & & & & $\mathrm{PBO}(\mathrm{N}=137)$ & $87.7 \pm 19.2$ & $\begin{array}{c}-0.9 \\
(-1.4,-0.4)\end{array}$ & - \\
\hline & & & & $\begin{array}{l}\text { DAPA } 2.5 \mathrm{mg} \\
(\mathrm{N}=137)\end{array}$ & $84.9 \pm 17.8$ & $\begin{array}{c}-2.2^{\dagger} \\
(-2.7,-1.8)\end{array}$ & - \\
\hline & & & & $\begin{array}{l}\text { DAPA } 5 \mathrm{mg} \\
(\mathrm{N}=137)\end{array}$ & $84.7 \pm 16.3$ & $\begin{array}{c}-3.0^{\dagger} \\
(-3.5,-2.6)\end{array}$ & - \\
\hline & & & & $\begin{array}{l}\text { DAPA } 10 \mathrm{mg} \\
\quad(\mathrm{N}=135)\end{array}$ & $86.3 \pm 17.5$ & $\begin{array}{c}-2.9^{\dagger} \\
(-3.3,-2.4)\end{array}$ & - \\
\hline & & & & ${ }^{*} \mathrm{p}<0.0001$ & & & \\
\hline & & & & ${ }^{\dagger}$ Significant after & sequential testing $\mathrm{p}$ & ocedure at $\alpha=0.05$ & \\
\hline \multirow{6}{*}{$\begin{array}{l}\text { Ferrannini } \\
\text { (2010) }\end{array}$} & \multirow{6}{*}{$\begin{array}{c}\text { Randomized } \\
\text { Double-blind } \\
\text { Parallel-group } \\
\text { Placebo- } \\
\text { controlled }\end{array}$} & \multirow{6}{*}{$\begin{array}{c}\text { Total: } 558 \\
\text { T2DM } \\
\text { (276M; 282F) } \\
\text { Main AM } \\
\text { Cohort: } \\
\text { 274 T2DM } \\
\text { (132M; } 142 \mathrm{~F})\end{array}$} & \multirow{2}{*}{$\begin{array}{c}\text { Diet/exercise } \\
\text { Placebo Lead-in } \\
\text { Period: } 2 \text { weeks } \\
\text { (1 week for patients } \\
\text { with enrollment } \\
\text { HbA1C } 10.1 \% \text { - } \\
\text { 12\%) }\end{array}$} & & $\begin{array}{c}\text { Baseline Mean } \pm \\
\text { SD }\end{array}$ & $\begin{array}{l}\text { C (\%) at } 24 \text { Week } \\
\text { Adjusted Mean } \\
\text { Change ( } 95 \% \\
\text { CI) }\end{array}$ & $\begin{array}{l}\text { Difference vs. } \\
\text { PBO }\end{array}$ \\
\hline & & & & PBO (N = 75) & $7.84 \pm 0.87$ & $\begin{array}{c}-0.23 \\
(-0.43,-0.02)\end{array}$ & - \\
\hline & & & $\begin{array}{c}\text { Double-blind } \\
\text { Placebo Controlled } \\
\text { Treatment Period: }\end{array}$ & $\begin{array}{l}\text { DAPA } 2.5 \mathrm{mg} \\
\quad(\mathrm{N}=65)\end{array}$ & $7.92 \pm 0.90$ & $\begin{array}{c}-0.58 \\
(-0.80,-0.36)\end{array}$ & - \\
\hline & & & $\begin{array}{l}24 \text { weeks } \\
\text { Patients with }\end{array}$ & $\begin{array}{l}\text { DAPA } 5 \mathrm{mg} \\
\quad(\mathrm{N}=64)\end{array}$ & $7.86 \pm 0.94$ & $\begin{array}{c}-0.77^{*} \\
(-0.99,-0.55)\end{array}$ & - \\
\hline & & & $\begin{array}{c}\text { HbA1C } 7.0 \%-10 \% \\
\text { entered main AM } \\
\text { cohort groups: }\end{array}$ & $\begin{array}{l}\text { DAPA } 10 \mathrm{mg} \\
\quad(\mathrm{N}=70)\end{array}$ & $8.01 \pm 0.96$ & $\begin{array}{c}-0.89^{* *} \\
(-1.10,-0.67)\end{array}$ & - \\
\hline & & & $\begin{array}{l}\text { Group A: placebo } \\
\text { daily in AM }\end{array}$ & $\begin{array}{l}{ }^{*} \mathrm{p}=0.0005 \text { vs. } \mathrm{P} \\
{ }^{* *} \mathrm{p}<0.0001 \text { vs. }\end{array}$ & & & \\
\hline
\end{tabular}




\section{Continued}

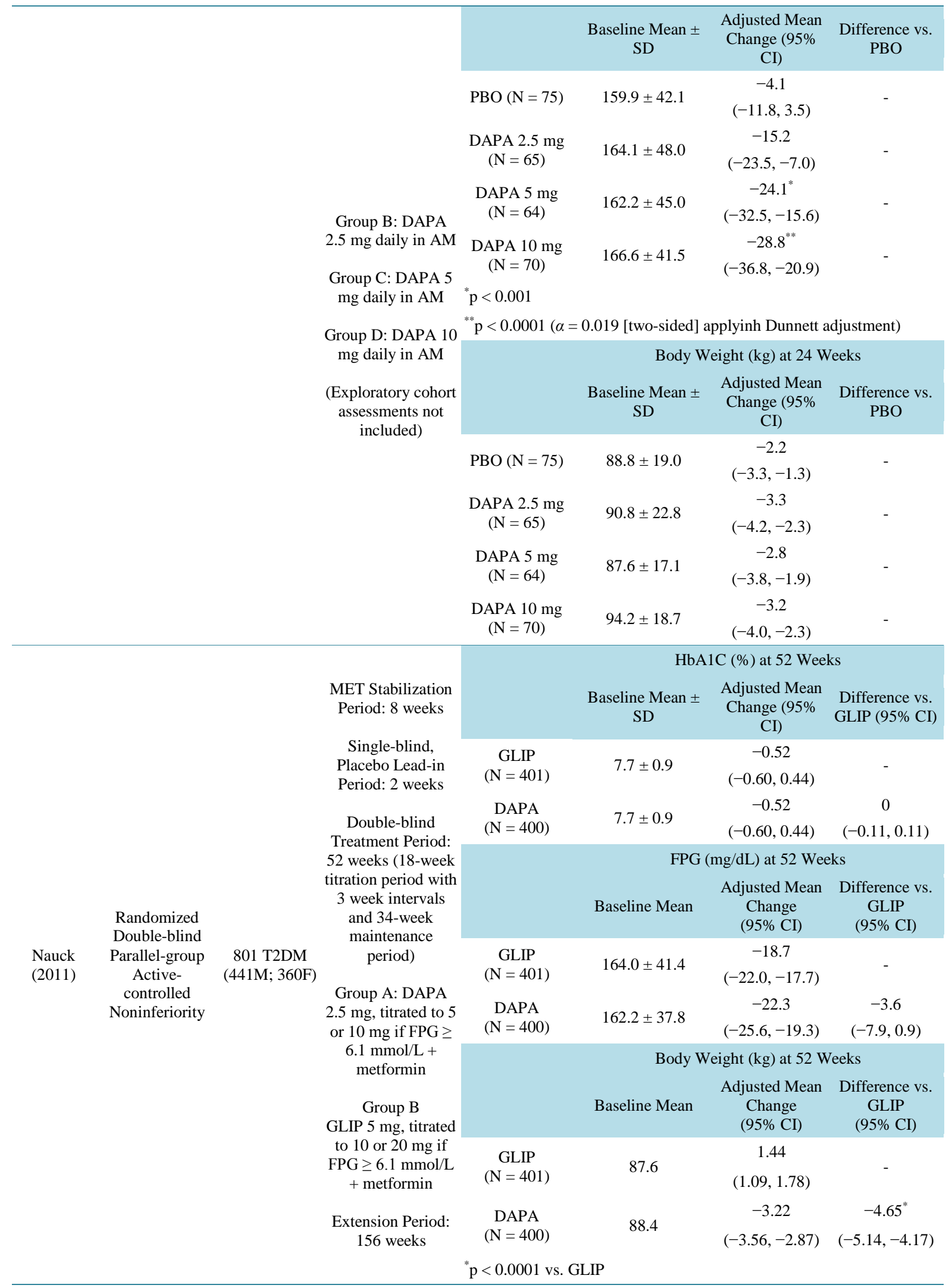




\section{Continued}

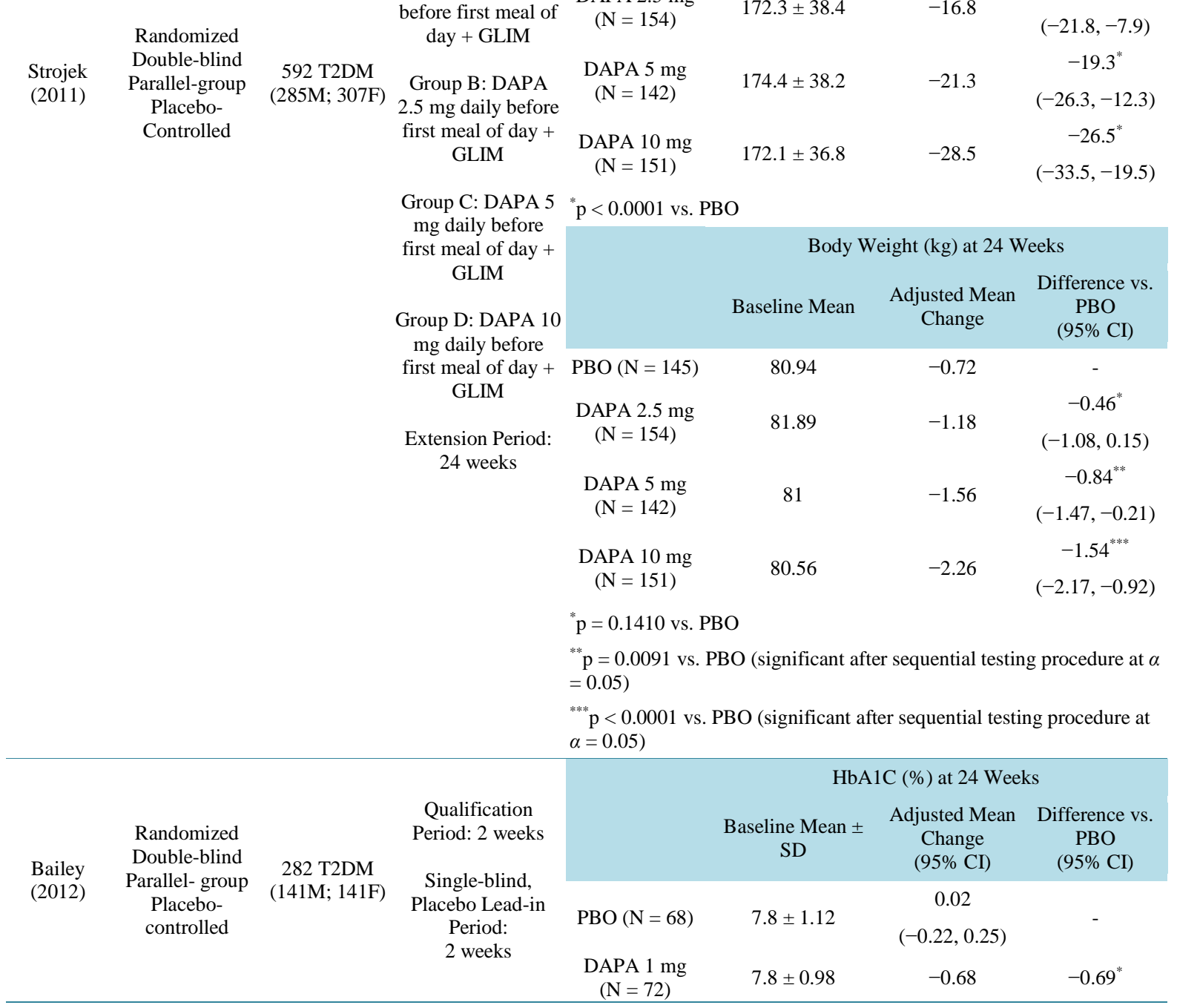

HbA1C (\%) at 24 Weeks

\begin{tabular}{cccc}
\multicolumn{4}{c}{ HbA1C (\%) at 24 Weeks } \\
& $\begin{array}{c}\text { Baseline Mean } \pm \\
\text { SD }\end{array}$ & $\begin{array}{c}\text { Adjusted Mean } \\
\text { Change }\end{array}$ & $\begin{array}{c}\text { Difference vs. } \\
\text { PBO } \\
(95 \% \text { CI })\end{array}$ \\
PBO (N = 145) & $8.15 \pm 0.74$ & -0.13 & - \\
DAPA 2.5 mg \\
$(\mathrm{N}=154)$ \\
$\begin{array}{c}\text { DAPA 5 mg } \\
(\mathrm{N}=142)\end{array}$ & $8.11 \pm 0.75$ & -0.58 & $-0.44^{*}$ \\
& $8.12 \pm 0.78$ & -0.63 & $-0.61,-0.27)$ \\
$\begin{array}{c}\text { DAPA 10 mg } \\
(\mathrm{N}=151)\end{array}$ & $8.07 \pm 0.79$ & -0.82 & $(-0.67,-0.32)$ \\
\end{tabular}

Qualification $\quad{ }^{*} \mathrm{p}<0.0001$ vs. PBO at $\alpha=0.019$ applying Dunnett’s adjustment Period: 1 week

Double-blind

Placebo-controlled Treatment Period: 24 weeks$$
\text { (BBO }
$$$$
\mathrm{PBO}
$$

$\mathrm{PB} O$

Group A: placebo fore first meal of

FPG $(\mathrm{mg} / \mathrm{dL})$ at 24 Weeks

$\begin{array}{lccc} & \begin{array}{c}\text { Baseline Mean } \pm \\ \text { SD }\end{array} & \begin{array}{c}\text { Adjusted Mean } \\ \text { Change }\end{array} & \begin{array}{c}\text { Difference vs. } \\ \text { PBO } \\ (95 \% \mathrm{CI})\end{array} \\ \begin{array}{cccc}\text { PBO (N = 145) } \\ \text { DAPA 2.5 mg } \\ (\mathrm{N}=154)\end{array} & 172.6 \pm 37.3 & -2 & - \\ & 172.3 \pm 38.4 & -16.8 & -15 \\ \begin{array}{c}\text { DAPA 5 mg } \\ \text { (N = 142) }\end{array} & 174.4 \pm 38.2 & -21.3 & -19.3^{*} \\ \begin{array}{c}\text { DAPA 10 mg } \\ (\mathrm{N}=151)\end{array} & 172.1 \pm 36.8 & -28.5 & (-26.3,-12.3) \\ \end{array}$

Group C: DAPA 5 * ${ }^{*}<0.0001$ vs. PBO

mg daily before

first meal of day +

roup D: DAPA 10

mg daily before

meal of day

tension Period:

DAPA $2.5 \mathrm{mg}$

DAPA $5 \mathrm{mg}$

APA $10 \mathrm{mg}$

$(\mathrm{N}=151)$

Baseline Mean

djusted Mean

PBO

$1.08,0.15)$

.47, -0.21)

$-1.54^{* * *}$

$(-2.17,-0.92)$

$\mathrm{p}=0.1410$ vs. $\mathrm{PBO}$

$0.0091 \mathrm{vs}$. PBO (significant after sequential testing procedure at $\alpha$

$\mathrm{p}<0.0001$ vs. PBO (significant after sequential testing procedure at $\alpha=0.05)$

$(\mathrm{N}=72)$ 


\section{Continued}

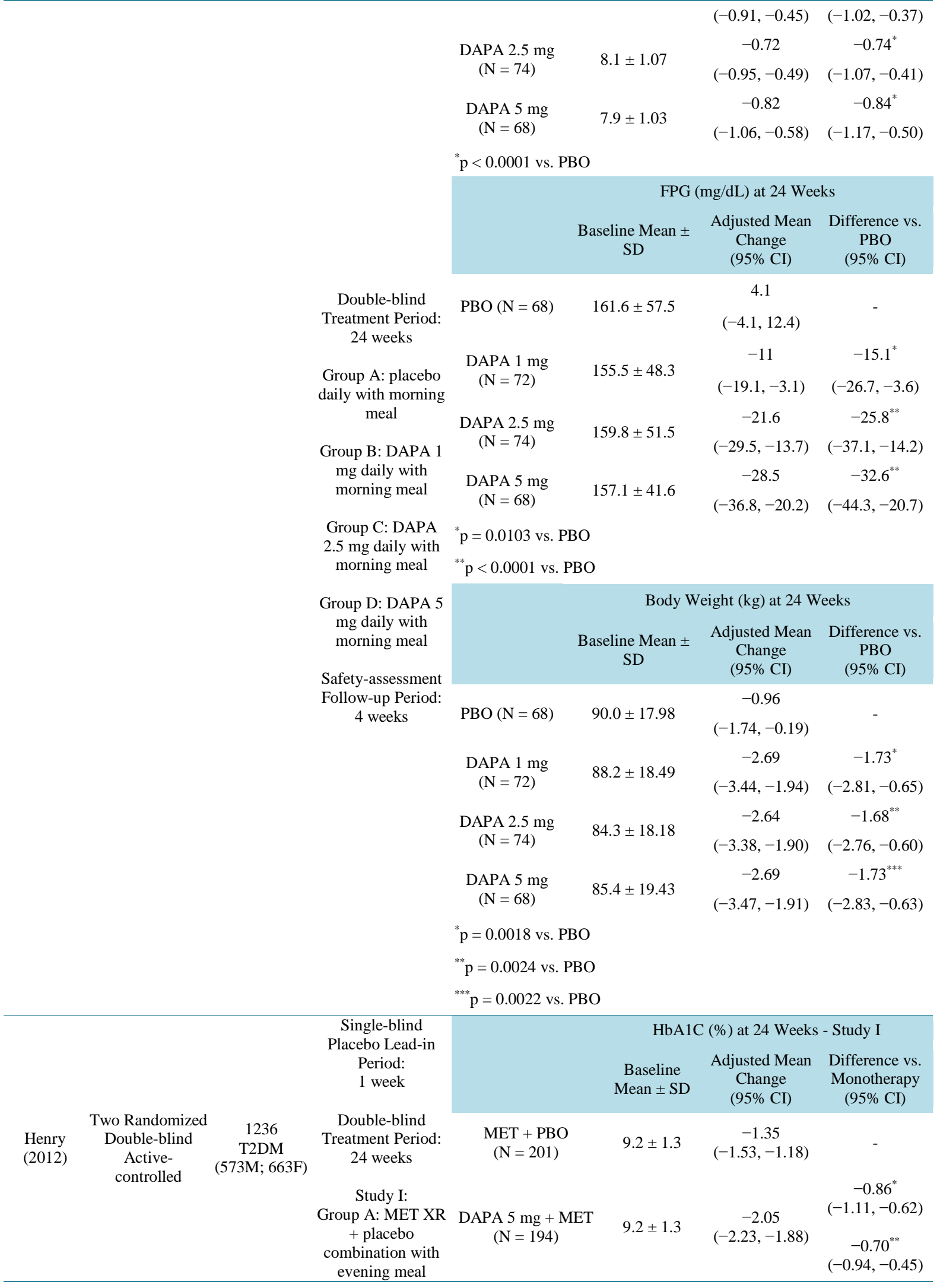




\section{Continued}

$\begin{array}{ccc}\text { DAPA } 5 \text { mg + PBO } & -1.19 \\ (\mathrm{~N}=203) & 9.1 \pm 1.4 & (-1.36,-1.02)\end{array}$

\begin{tabular}{cccc} 
& \multicolumn{3}{c}{ HbA1C (\%) at 24 Weeks - Study II } \\
& $\begin{array}{c}\text { Baseline } \\
\text { Mean } \pm \text { SD }\end{array}$ & $\begin{array}{c}\text { Adjusted Mean } \\
\text { Change } \\
(95 \% \text { CI })\end{array}$ & $\begin{array}{c}\text { Difference vs. } \\
\text { Monotherapy } \\
(95 \% \text { CI })\end{array}$ \\
MET + PBO & $9.1 \pm 1.3$ & -1.44 & - \\
$(\mathrm{N}=208)$ & & $(-1.59,-1.29)$ & \\
& & & $-0.53^{*}$ \\
DAPA 10 mg + & & -1.98 & $(-0.74,-0.32)$ \\
MET & $9.1 \pm 1.3$ & $(-2.13,-1.83)$ & $-0.54^{* *}$ \\
(N = 211) & & & $(-0.75,-0.33)$ \\
& & & - \\
DAPA 10 mg + PBO & $9.1 \pm 1.3$ & $(-1.59,-1.31)$ & - \\
$(\mathrm{N}=219)$ & & &
\end{tabular}

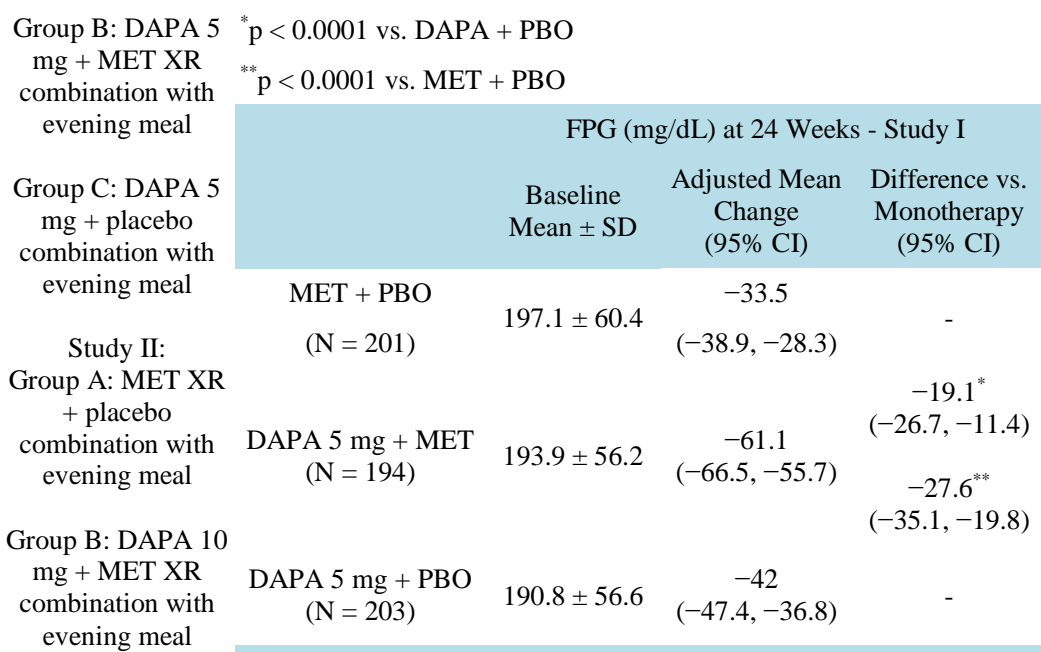
Group C: DAPA 10
$\mathrm{mg}+$ placebo combination with evening meal

\begin{tabular}{|c|c|c|c|}
\hline & \multicolumn{3}{|c|}{ FPG (mg/dL) at 24 Weeks - Study II } \\
\hline & $\begin{array}{c}\text { Baseline } \\
\text { Mean } \pm \text { SD }\end{array}$ & $\begin{array}{l}\text { Adjusted Mean } \\
\text { Change } \\
(95 \% \text { CI) }\end{array}$ & $\begin{array}{c}\text { Difference vs. } \\
\text { Monotherapy } \\
(95 \% \text { CI })\end{array}$ \\
\hline $\begin{array}{l}\mathrm{MET}+\mathrm{PBO} \\
(\mathrm{N}=208)\end{array}$ & $190.5 \pm 54.1$ & $\begin{array}{c}-34.8 \\
(-39.8,-29.7)\end{array}$ & - \\
\hline $\begin{array}{c}\text { DAPA } 10 \mathrm{mg}+ \\
\text { MET } \\
(\mathrm{N}=211)\end{array}$ & $189.5 \pm 58.0$ & $\begin{array}{c}-60.4 \\
(-65.2,-55.3)\end{array}$ & $\begin{array}{c}-13.9^{*} \\
(-20.9,-7.0) \\
-25.6^{* *} \\
(-32.6,-18.6)\end{array}$ \\
\hline $\begin{array}{l}\text { DAPA } 10 \mathrm{mg}+\text { PBO } \\
\quad(\mathrm{N}=219)\end{array}$ & $198.0 \pm 61.8$ & $\begin{array}{c}-46.5 \\
(-51.4,-41.4)\end{array}$ & - \\
\hline
\end{tabular}

${ }^{*} \mathrm{p}<0.0001$ vs. DAPA + PBO (after sequential testing procedure at $\alpha=$ $0.05)$

${ }^{* *} \mathrm{p}<0.0001$ vs. MET + PBO (after sequential testing procedure at $\alpha=$ $0.05)$

Body Weight (kg) at 24 Weeks - Study I

$\begin{array}{ccc}\text { Baseline } & \begin{array}{c}\text { Adjusted Mean } \\ \text { Change } \\ \text { Mean } \pm \text { SD }\end{array} & \begin{array}{c}\text { Difference vs. } \\ \text { Monotherapy } \\ (95 \% \text { CI })\end{array} \\ & & \\ (95 \% \text { CI })\end{array}$




\section{Continued}

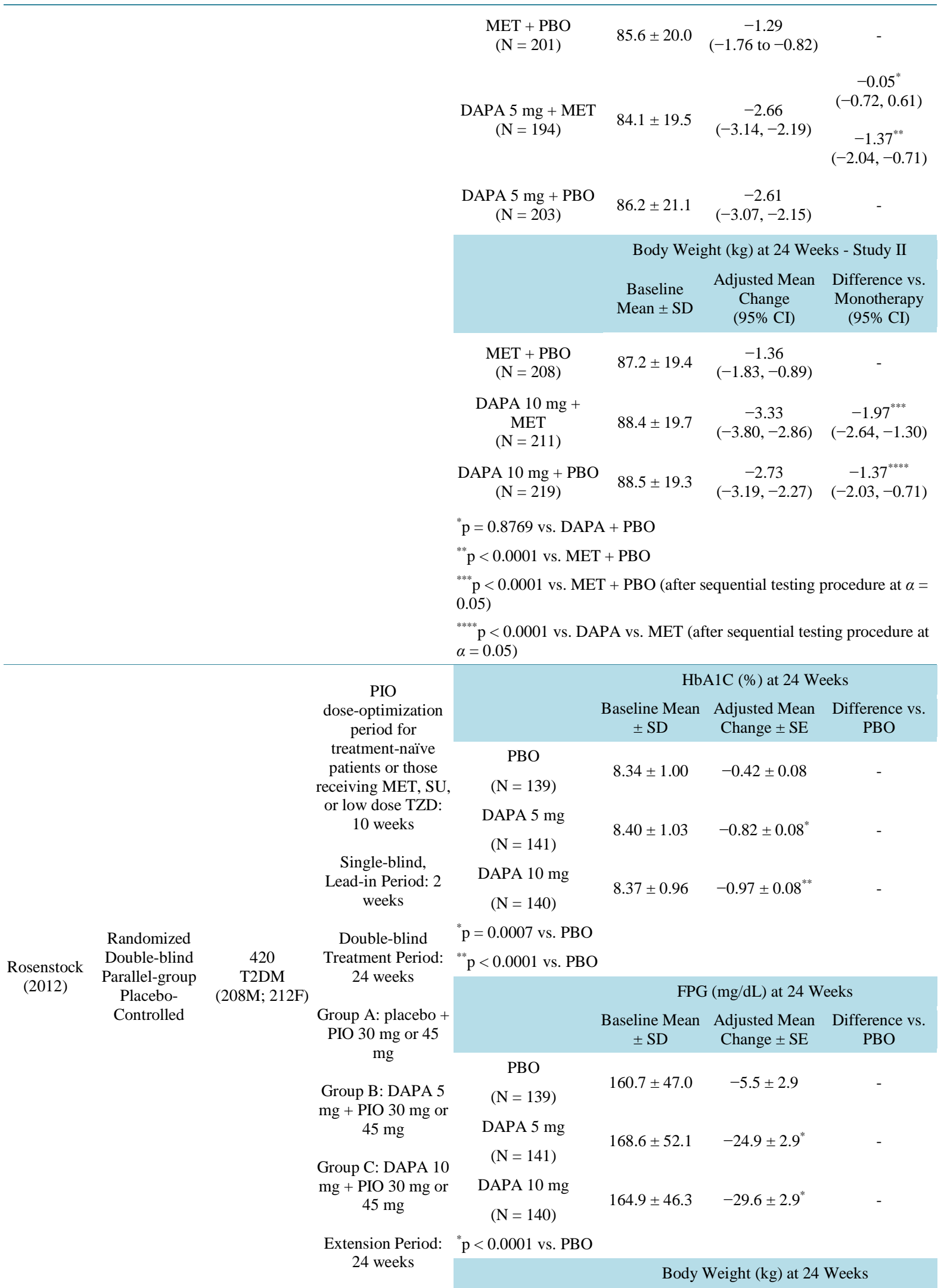




\section{Continued}

\begin{tabular}{|c|c|c|c|c|c|c|c|}
\hline & & & & & $\begin{array}{c}\text { Baseline Mean } \\
\pm \text { SD }\end{array}$ & $\begin{array}{l}\text { Adjusted Mean } \\
\text { Change } \pm \text { SE }\end{array}$ & $\begin{array}{c}\text { Difference vs. } \\
\text { PBO }\end{array}$ \\
\hline & & & & $\begin{array}{c}\text { PBO } \\
(\mathrm{N}=139)\end{array}$ & $86.4 \pm 21.3$ & $1.64 \pm 0.28$ & - \\
\hline & & & & $\begin{array}{l}\text { DAPA } 5 \mathrm{mg} \\
(\mathrm{N}=141)\end{array}$ & $87.8 \pm 20.7$ & $0.09 \pm 0.28^{*}$ & - \\
\hline & & & & $\begin{array}{c}\text { DAPA } 10 \mathrm{mg} \\
(\mathrm{N}=140)\end{array}$ & $84.8 \pm 22.2$ & $-0.14 \pm 0.28^{*}$ & - \\
\hline & & & & ${ }^{*} \mathrm{p}<0.0001$ vs. $\mathrm{PBO}$ & & & \\
\hline & & & & & $\mathrm{Hb}$ & $\mathrm{A} 1 \mathrm{C}(\%)$ at $48 \mathrm{~W}$ & eeks \\
\hline & & & & & $\begin{array}{l}\text { Baseline Mean } \\
\quad \pm \text { SD }\end{array}$ & $\begin{array}{l}\text { Adjusted Mean } \\
\text { Change } \\
\text { (95\% CI) }\end{array}$ & $\begin{array}{c}\text { Difference vs. } \\
\text { PBO }\end{array}$ \\
\hline & & & & $\begin{array}{c}\text { PBO } \\
(\mathrm{N}=139)\end{array}$ & $8.34 \pm 1.00$ & $\begin{array}{c}-0.54 \\
(-0.70,-0.38)\end{array}$ & - \\
\hline & & & & $\begin{array}{l}\text { DAPA } 5 \text { mg } \\
(\mathrm{N}=141)\end{array}$ & $8.40 \pm 1.03$ & $\begin{array}{c}-0.95 \\
(-1.10,-0.80)\end{array}$ & - \\
\hline & & & & $\begin{array}{l}\text { DAPA } 10 \mathrm{mg} \\
(\mathrm{N}=140)\end{array}$ & $8.37 \pm 0.96$ & $\begin{array}{c}-1.21 \\
(-1.36,-1.06)\end{array}$ & - \\
\hline & & & & & FPG & $(\mathrm{mg} / \mathrm{dL})$ at $48 \mathrm{~W}$ & eeks \\
\hline & & & & & $\begin{array}{c}\text { Baseline Mean } \\
\pm \text { SD }\end{array}$ & $\begin{array}{l}\text { Adjusted Mean } \\
\text { Change } \\
(95 \% \text { CI })\end{array}$ & $\begin{array}{c}\text { Difference vs. } \\
\text { PBO }\end{array}$ \\
\hline & & & & $\begin{array}{c}\text { PBO } \\
(\mathrm{N}=139)\end{array}$ & $160.7 \pm 47.0$ & $\begin{array}{c}-13.1 \\
(-20.2,-6.0)\end{array}$ & - \\
\hline & & & & $\begin{array}{l}\text { DAPA } 5 \text { mg } \\
(\mathrm{N}=141)\end{array}$ & $168.6 \pm 52.1$ & $\begin{array}{c}-22.8 \\
(-29.1 \text { to }-16.4)\end{array}$ & - \\
\hline & & & & $\begin{array}{l}\text { DAPA } 10 \mathrm{mg} \\
(\mathrm{N}=140)\end{array}$ & $164.9 \pm 46.3$ & $\begin{array}{c}-33.1 \\
(-39.0,-27.2)\end{array}$ & - \\
\hline & & & & & Body & Weight $(\mathrm{kg})$ at 48 & Weeks \\
\hline & & & & & $\begin{array}{l}\text { Baseline Mean } \\
\quad \pm \text { SD }\end{array}$ & $\begin{array}{l}\text { Adjusted Mean } \\
\text { Change } \\
\text { (95\% CI) }\end{array}$ & $\begin{array}{c}\text { Difference vs. } \\
\text { PBO }\end{array}$ \\
\hline & & & & $\begin{array}{c}\text { PBO } \\
(\mathrm{N}=139)\end{array}$ & $86.4 \pm 21.3$ & $\begin{array}{c}2.99 \\
(2.19,3.79)\end{array}$ & - \\
\hline & & & & $\begin{array}{l}\text { DAPA } 5 \mathrm{mg} \\
(\mathrm{N}=141)\end{array}$ & $87.8 \pm 20.7$ & $\begin{array}{c}1.35 \\
(0.61,2.09)\end{array}$ & - \\
\hline & & & & $\begin{array}{l}\text { DAPA } 10 \mathrm{mg} \\
(\mathrm{N}=140)\end{array}$ & $84.8 \pm 22.2$ & $\begin{array}{c}0.69 \\
(-0.03,1.41)\end{array}$ & - \\
\hline & & & & & $\mathrm{Hb}$ & $\mathrm{A} 1 \mathrm{C}(\%)$ at $24 \mathrm{~W}$ & eeks \\
\hline & $\begin{array}{l}\text { Randomized } \\
\text { Double-blind }\end{array}$ & & $\begin{array}{c}\text { Pre-enrollment } \\
\text { OAD/ } \\
\text { Insulin Stabilization } \\
\text { Period: at least } 8\end{array}$ & & $\begin{array}{c}\text { Baseline Mean } \\
\pm \text { SD }\end{array}$ & $\begin{array}{l}\text { Adjusted Mean } \\
\text { Change }\end{array}$ & $\begin{array}{c}\text { Difference vs. } \\
\text { PBO } \\
(95 \% \mathrm{CI})\end{array}$ \\
\hline$(2012)$ & $\begin{array}{l}\text { Parallel-group } \\
\text { Placebo- } \\
\text { controlled }\end{array}$ & (382M; 418F) & weeks & $\begin{array}{c}\text { PBO } \\
(\mathrm{N}=193)\end{array}$ & $8.47 \pm 0.77$ & -0.39 & - \\
\hline & & & 2 weeks & $\begin{array}{l}\text { DAPA } 2.5 \mathrm{mg} \\
(\mathrm{N}=202)\end{array}$ & $8.46 \pm 0.78$ & -0.79 & $\begin{array}{c}-0.40^{*} \\
(-0.54,-0.25)\end{array}$ \\
\hline
\end{tabular}




\section{Continued}

Double-blind

Placebo-controlled

Treatment Period: 24 weeks

(with open-label insulin/existing OAD therapies)

Group A: placebo + insulin

Group B: DAPA

$2.5 \mathrm{mg}$ daily + insulin

Group C: DAPA 5 mg daily + insulin

Group D: DAPA 10

mg daily + insulin

Double-blind

Extension Period I:

24 weeks

Double-blind Extension Period II: 56 weeks

\begin{tabular}{|c|c|c|c|}
\hline $\begin{array}{l}\text { DAPA } 5 \mathrm{mg} \\
(\mathrm{N}=211)\end{array}$ & $8.62 \pm 0.89$ & -0.89 & $\begin{array}{c}-0.49^{*} \\
(-0.65,-0.34)\end{array}$ \\
\hline $\begin{array}{l}\text { DAPA } 10 \mathrm{mg} \\
(\mathrm{N}=194)\end{array}$ & $8.57 \pm 0.82$ & -0.96 & $\begin{array}{c}-0.57^{*} \\
(-0.72,-0.42)\end{array}$ \\
\hline
\end{tabular}

${ }^{*} \mathrm{p}<0.001$ vs. $\mathrm{PBO}$

FPG (mg/dL) at 24 Weeks

$\begin{array}{ccc}\text { Baseline Mean } & \begin{array}{c}\text { Adjusted Mean } \\ \text { Change }\end{array} & \text { Difference vs. } \\ \text { SD } & \text { (95\% CI }) & \text { PBO }\end{array}$

\section{PBO}

$(\mathrm{N}=193)$

DAPA $2.5 \mathrm{mg}$

$(\mathrm{N}=202)$

DAPA $5 \mathrm{mg}$

$(\mathrm{N}=211)$

DAPA $10 \mathrm{mg}$

$(\mathrm{N}=194)$

$<0.001$ vs. baseline

\begin{tabular}{|c|c|c|c|}
\hline & \multicolumn{3}{|c|}{ Body Weight (kg) at 24 Weeks } \\
\hline & $\begin{array}{c}\text { Baseline Mean } \\
\pm \text { SD }\end{array}$ & $\begin{array}{l}\text { Adjusted Mean } \\
\text { Change }\end{array}$ & $\begin{array}{c}\text { Difference vs. } \\
\text { PBO } \\
(95 \% \text { CI })\end{array}$ \\
\hline $\begin{array}{c}\text { PBO } \\
(\mathrm{N}=193)\end{array}$ & $94.5 \pm 19.8$ & 0.43 & - \\
\hline $\begin{array}{c}\text { DAPA } 2.5 \mathrm{mg} \\
\quad(\mathrm{N}=202)\end{array}$ & $93.0 \pm 16.7$ & -0.92 & $\begin{array}{c}-1.35^{*} \\
(-1.90,-0.80)\end{array}$ \\
\hline $\begin{array}{l}\text { DAPA } 5 \mathrm{mg} \\
(\mathrm{N}=211)\end{array}$ & $93.3 \pm 17.4$ & -1 & $\begin{array}{c}-1.42^{*} \\
(-1.97,-0.88)\end{array}$ \\
\hline $\begin{array}{c}\text { DAPA } 10 \mathrm{mg} \\
\quad(\mathrm{N}=194)\end{array}$ & $94.5 \pm 16.8$ & -1.61 & $\begin{array}{c}-2.04^{*} \\
(-2.59,-1.48)\end{array}$ \\
\hline
\end{tabular}

${ }^{*} \mathrm{p}<0.001$ vs. $\mathrm{PBO}$

\begin{tabular}{cccc}
\multicolumn{4}{c}{ HbA1C (\%) at 48 Weeks } \\
& $\begin{array}{c}\text { Baseline Mean } \\
\pm \text { SD }\end{array}$ & $\begin{array}{c}\text { Adjusted Mean } \\
\text { Change }\end{array}$ & $\begin{array}{c}\text { Difference vs. } \\
\text { PBO } \\
(95 \% \text { CI })\end{array}$ \\
PBO & $8.47 \pm 0.77$ & -0.47 & - \\
$(\mathrm{N}=193)$ & & & $-0.32^{*}$ \\
DAPA 2.5 mg & $8.46 \pm 0.78$ & -0.79 & $(-0.48,-0.16)$ \\
$(\mathrm{N}=$ 202) & & & $-0.49^{*}$ \\
DAPA 5 mg & $8.62 \pm 0.89$ & -0.96 & $(-0.65,-0.33)$ \\
$(\mathrm{N}=211)$ & & & $-0.54^{*}$ \\
DAPA 10 mg & $8.57 \pm 0.82$ & -1.01 & $(-0.70,-0.38)$ \\
$(\mathrm{N}=194)$ & & &
\end{tabular}

${ }^{*} \mathrm{p}<0.001$ vs. $\mathrm{PBO}$ 


\section{Continued}

\begin{tabular}{|c|c|c|c|c|c|c|c|}
\hline & & & & & $\begin{array}{l}\text { Baseline Mean } \\
\quad \pm \text { SD }\end{array}$ & $\begin{array}{l}\text { Adjusted Mean } \\
\text { Change } \\
\text { (95\% CI) }\end{array}$ & $\begin{array}{c}\text { Difference vs. } \\
\text { PBO }\end{array}$ \\
\hline & & & & $\begin{array}{c}\text { PBO } \\
(\mathrm{N}=193)\end{array}$ & $170.6 \pm 57.2$ & - & - \\
\hline & & & & $\begin{array}{l}\text { DAPA } 2.5 \mathrm{mg} \\
\quad(\mathrm{N}=202)\end{array}$ & $180.1 \pm 59.9$ & $\begin{array}{c}-12.43^{*} \\
(-23.1,-2.0)\end{array}$ & - \\
\hline & & & & $\begin{array}{l}\text { DAPA } 5 \mathrm{mg} \\
\quad(\mathrm{N}=211)\end{array}$ & $185.4 \pm 58.7$ & $\begin{array}{c}-16.2^{*} \\
(-26.7,-6.0)\end{array}$ & - \\
\hline & & & & $\begin{array}{c}\text { DAPA } 10 \mathrm{mg} \\
(\mathrm{N}=194)\end{array}$ & $173.1 \pm 54.9$ & $\begin{array}{c}-16.94^{*} \\
(-27.6,-6.5)\end{array}$ & - \\
\hline & & & & ${ }^{*} \mathrm{p}<0.001$ vs. baseline & & & \\
\hline & & & & & Body & Weight $(\mathrm{kg})$ at 48 & Weeks \\
\hline & & & & & $\begin{array}{l}\text { Baseline Mean } \\
\quad \pm \text { SD }\end{array}$ & $\begin{array}{l}\text { Adjusted Mean } \\
\text { Change }\end{array}$ & $\begin{array}{c}\text { Difference vs. } \\
\text { PBO } \\
(95 \% \text { CI) }\end{array}$ \\
\hline & & & & $\begin{array}{c}\text { PBO } \\
(\mathrm{N}=193)\end{array}$ & $94.5 \pm 19.8$ & 0.82 & - \\
\hline & & & & $\begin{array}{l}\text { DAPA } 2.5 \mathrm{mg} \\
\quad(\mathrm{N}=202)\end{array}$ & $93.0 \pm 16.7$ & -0.96 & $\begin{array}{c}-1.78^{*} \\
(-2.53,-1.03)\end{array}$ \\
\hline & & & & $\begin{array}{l}\text { DAPA } 5 \mathrm{mg} \\
(\mathrm{N}=211)\end{array}$ & $93.3 \pm 17.4$ & -1 & $\begin{array}{c}-1.82^{*} \\
(-2.56,-1.07)\end{array}$ \\
\hline & & & & $\begin{array}{c}\text { DAPA } 10 \mathrm{mg} \\
(\mathrm{N}=194)\end{array}$ & $94.5 \pm 16.8$ & -1.61 & $\begin{array}{c}-2.43^{*} \\
(-3.18,-1.68)\end{array}$ \\
\hline & & & & ${ }^{*} \mathrm{p}<0.001$ vs. $\mathrm{PBO}$ & & & \\
\hline \multirow{10}{*}{$\begin{array}{l}\text { Bailey } \\
\text { (2013) }\end{array}$} & \multirow{10}{*}{$\begin{array}{c}\text { Randomized } \\
\text { Double-blind } \\
\text { Parallel Group } \\
\text { Placebo- } \\
\text { Controlled }\end{array}$} & \multirow{10}{*}{$\begin{array}{c}546 \text { T2DM } \\
\text { (292M; 254F) }\end{array}$} & \multirow[b]{3}{*}{$\begin{array}{l}\text { Double-blind } \\
\text { Extension } \\
\text { Treatment Period: } \\
78 \text { weeks } \\
\text { (All groups on } \\
\text { stable MET dose) }\end{array}$} & & \multicolumn{3}{|c|}{ HbA1C (\%) at 102 Weeks } \\
\hline & & & & & $\begin{array}{l}\text { Baseline Mean } \\
\quad \pm \text { SD }\end{array}$ & $\begin{array}{l}\text { Adjusted Mean } \\
\text { Change } \\
(95 \% \text { CI })\end{array}$ & $\begin{array}{c}\text { Difference vs. } \\
\text { PBO } \\
(95 \% \mathrm{CI})\end{array}$ \\
\hline & & & & $\begin{array}{c}\text { PBO } \\
(\mathrm{N}=137) \\
\text { DAPA } 2.5 \mathrm{mg} \\
(\mathrm{N}=137)\end{array}$ & $7.99 \pm 0.90$ & $\begin{array}{c}0.02 \\
(-0.20,0.23) \\
-0.48 \\
(-0.68,-0.29)\end{array}$ & $\begin{array}{c}- \\
-0.50^{*} \\
(-0.79,-0.21)\end{array}$ \\
\hline & & & $\begin{array}{c}\text { Group A: placebo + } \\
\text { MET daily in AM }\end{array}$ & $\begin{array}{l}\text { DAPA } 5 \text { mg } \\
(\mathrm{N}=137)\end{array}$ & $8.17 \pm 0.96$ & $\begin{array}{c}-0.58 \\
(-0.77,-0.39)\end{array}$ & $\begin{array}{c}-0.60^{* *} \\
(-0.89,-0.31)\end{array}$ \\
\hline & & & $\begin{array}{c}\text { Group B: DAPA } \\
2.5 \mathrm{mg}+\mathrm{MET} \\
\text { daily in AM }\end{array}$ & $\begin{array}{c}\text { DAPA } 10 \mathrm{mg} \\
(\mathrm{N}=135)\end{array}$ & $7.92 \pm 0.82$ & $\begin{array}{c}-0.78 \\
(-0.97,-0.60)\end{array}$ & $\begin{array}{c}-0.80^{* *} \\
(-1.08,-0.52)\end{array}$ \\
\hline & & & \multirow{2}{*}{$\begin{array}{c}\text { Group C: DAPA } 5 \\
\text { mg + MET daily in } \\
\text { AM }\end{array}$} & $\begin{array}{l}{ }^{*} \mathrm{p}=0.0008 \\
{ }^{* *} \mathrm{p}<0.0001 \text { vs. } \mathrm{PBO}\end{array}$ & & & \\
\hline & & & & & \multicolumn{3}{|c|}{ FPG (mg/dL) at 102 Weeks } \\
\hline & & & \multirow[t]{3}{*}{$\begin{array}{c}\text { Group D: DAPA } 10 \\
\text { mg + MET daily in } \\
\text { AM }\end{array}$} & & $\begin{array}{l}\text { Baseline Mean } \\
\quad \pm \text { SD }\end{array}$ & $\begin{array}{l}\text { Adjusted Mean } \\
\text { Change } \\
(95 \% \text { CI })\end{array}$ & $\begin{array}{c}\text { Difference vs. } \\
\text { PBO } \\
(95 \% \text { CI })\end{array}$ \\
\hline & & & & $\begin{array}{c}\text { PBO } \\
(\mathrm{N}=137)\end{array}$ & $165.6 \pm 46.5$ & $\begin{array}{c}-10.5 \\
(-17.5,-3.4)\end{array}$ & - \\
\hline & & & & DAPA $2.5 \mathrm{mg}$ & $161.4 \pm 43.1$ & -19.3 & $-8.8^{*}$ \\
\hline
\end{tabular}




\section{Continued}

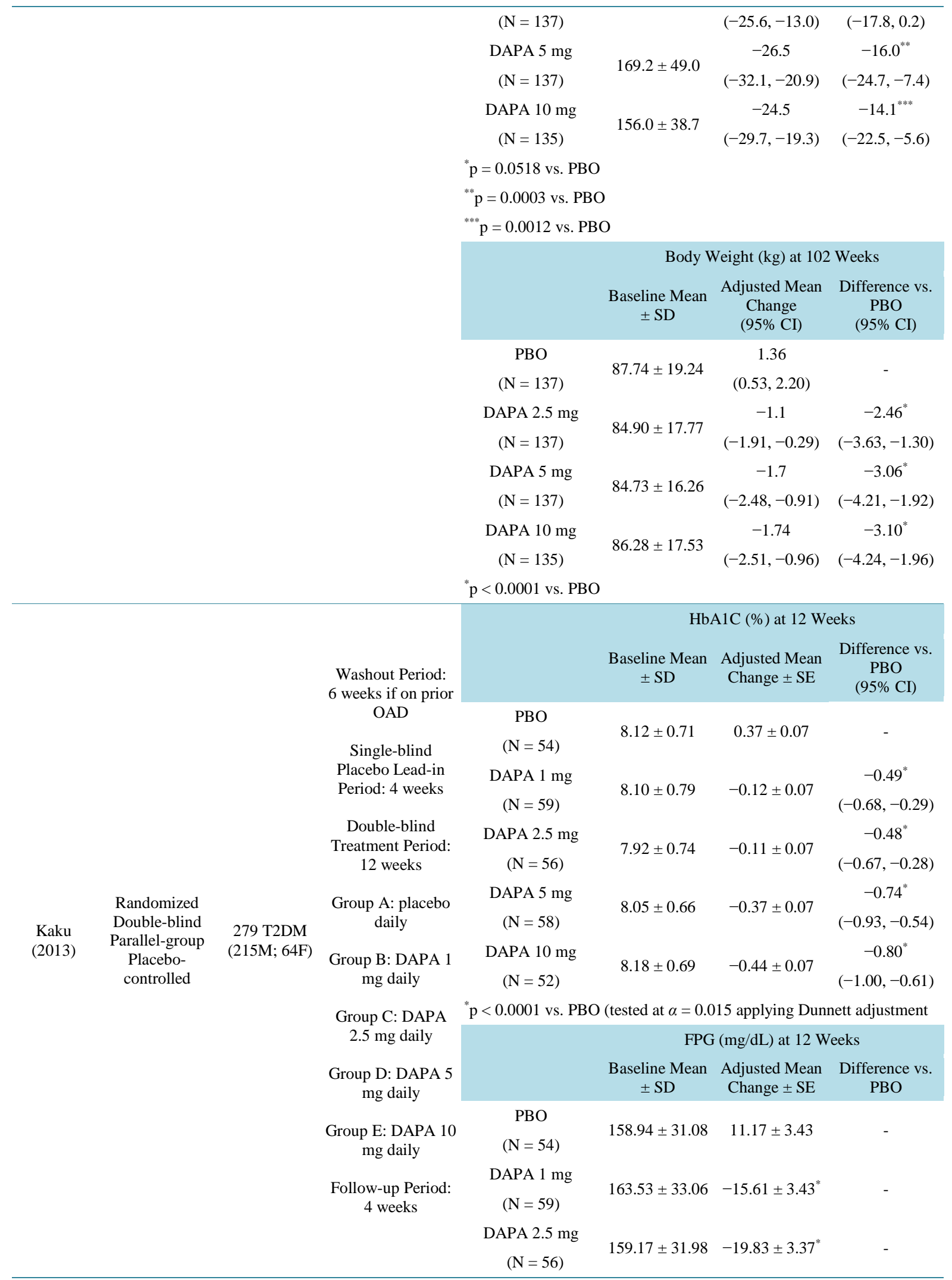




\begin{tabular}{|c|c|c|c|}
\hline $\begin{array}{l}\text { DAPA } 5 \mathrm{mg} \\
(\mathrm{N}=58)\end{array}$ & $164.49 \pm 23.56$ & $-23.51 \pm 3.43^{*}$ & - \\
\hline $\begin{array}{l}\text { DAPA } 10 \mathrm{mg} \\
\qquad(\mathrm{N}=52)\end{array}$ & $163.36 \pm 29.74$ & $-31.94 \pm 3.57^{*}$ & - \\
\hline $\mathrm{p} p<0.0001$ vs. $\mathrm{PB}$ & & & \\
\hline & Body & Neight $(\mathrm{kg})$ at 12 & Weeks \\
\hline & $\begin{array}{c}\text { Baseline Mean } \\
\pm \text { SD }\end{array}$ & $\begin{array}{c}\text { Adjusted Mean } \\
\text { Change } \pm \text { SE }\end{array}$ & $\begin{array}{c}\text { Difference vs. } \\
\text { PBO }\end{array}$ \\
\hline $\begin{array}{c}\text { PBO } \\
(n=54)\end{array}$ & $68.88 \pm 14.94$ & $-0.05 \pm 0.19$ & - \\
\hline $\begin{array}{l}\text { DAPA } 1 \mathrm{mg} \\
\qquad(\mathrm{n}=59)\end{array}$ & $68.40 \pm 11.04$ & $-1.25 \pm 0.18^{*}$ & - \\
\hline $\begin{array}{c}\text { DAPA } 2.5 \mathrm{mg} \\
\quad(\mathrm{n}=56)\end{array}$ & $66.61 \pm 14.29$ & $-1.24 \pm 0.18^{*}$ & - \\
\hline $\begin{array}{l}\text { DAPA } 5 \mathrm{mg} \\
\quad(\mathrm{n}=58)\end{array}$ & $68.92 \pm 12.43$ & $-2.06 \pm 0.18^{*}$ & - \\
\hline $\begin{array}{c}\text { DAPA } 10 \mathrm{mg} \\
\quad(\mathrm{n}=52)\end{array}$ & $70.35 \pm 17.48$ & $-1.91 \pm 0.19^{*}$ & - \\
\hline & ${ }^{*} \mathrm{p}<0.0001$ & Is. PBO & \\
\hline
\end{tabular}

95\% CI = 95\% confidence interval; AHA = antihyperglycemic agent; AM = morning; DAPA = dapagliflozin; F = Female; FPG = Fasting Plasma Glucose; GLIM = glimepiride; GLIP = glipizide; HbA1C = hemoglobin A1C; $\mathrm{M}=$ Male; MET = metformin; PBO = placebo; SD = standard deviation; SE = standard error; SITA = sitagliptin; SU = sulfonylurea; T2DM = Type 2 Diabetes Mellitus Patients; TZD = thiazolidinedione.

with normal renal function. On the other hand, patients with mild and severe renal impairment have approximately 20\% higher peak plasma concentrations. Renal function should be evaluated prior to and throughout empagliflozin treatment. Use of empagliflozin should be avoided in patients with eGFR's of $<45 \mathrm{~mL} / \mathrm{min} /$ $1.73 \mathrm{~m}^{2}$. Mild to severe hepatic impairment may also result in AUC and $\mathrm{C}_{\max }$ elevations, but dose adjustments are not warranted [3].

Two pivotal trials evaluating the efficacy of empagliflozin in T2DM patients over 12 weeks were identified and summarized in Table 3. One study [30] assessed empagliflozin as monotherapy and another [31] as add-on therapy to metformin. Doses ranged from $1 \mathrm{mg}$ to $50 \mathrm{mg}$. Significant reductions in HbA1C, FPG, and weight were observed in the $5 \mathrm{mg}, 10 \mathrm{mg}, 25 \mathrm{mg}$, and $50 \mathrm{mg}$ empagliflozin groups when compared to placebo [30] [31]. Both studies reported greater proportions of empagliflozin groups reaching $\mathrm{HbA} 1 \mathrm{Cs} \leq 7 \%$ compared to placebo (30\% - 45\% of empagliflozin $5 \mathrm{mg}$ and $25 \mathrm{mg}$ versus 22\% of placebo [30] and 35.7\% - 38.0\% of empagliflozin $10 \mathrm{mg}, 25 \mathrm{mg}$, and $50 \mathrm{mg}$ versus $15.5 \%$ of placebo [31]). Similar reductions were observed with sitagliptin 100 mg (33.8\%) compared to placebo (15.5\%) [31].

Overall, adverse events were similar among empagliflozin, placebo, and open-label agent groups. Pollakiuria, thirst, nasopharyngitis, urinary tract infections, and genital infections were the most common adverse events reported by empagliflozin subjects. Although the incidence of UTIs was comparable among study groups within each trial, an increased number of genital infections occurred in the empagliflozin groups. Hypoglycemic episodes among treatment and placebo groups were not significantly different. Additionally, both studies reported a trend toward a potential dose-related increase in hematocrit in the empagliflozin groups ( $0.6 \%-2.5 \%)$ [30] [31]. Blood pressure (systolic and diastolic) changes trended toward dose-dependent decreases in the empagliflozin (5 $\mathrm{mg}, 10 \mathrm{mg}$, and $25 \mathrm{mg}$ ) versus placebo groups but were not statistically significant. The greatest change occurred in the empagliflozin $25 \mathrm{mg}$ group [31].

\subsection{Other SGLT2s}

Although canagliflozin, dapagliflozin, and empagliflozin are the only SGLT2 inhibitors on the US market, several other candidates are currently in development. These include ipragliflozin, tofogliflozin, and ertugliflozin. 
Table 3. Empagliflozin trials.

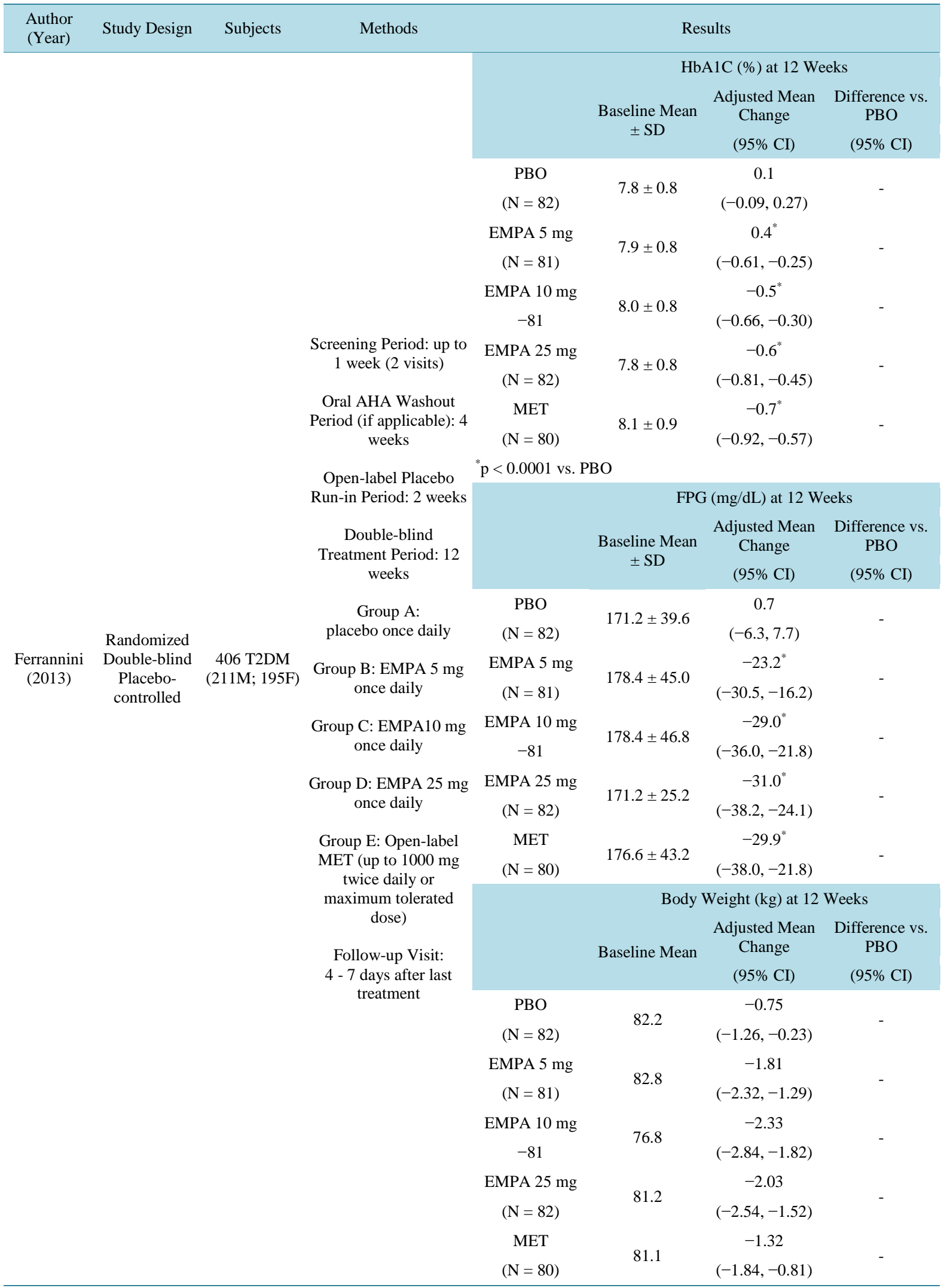




\section{Continued}

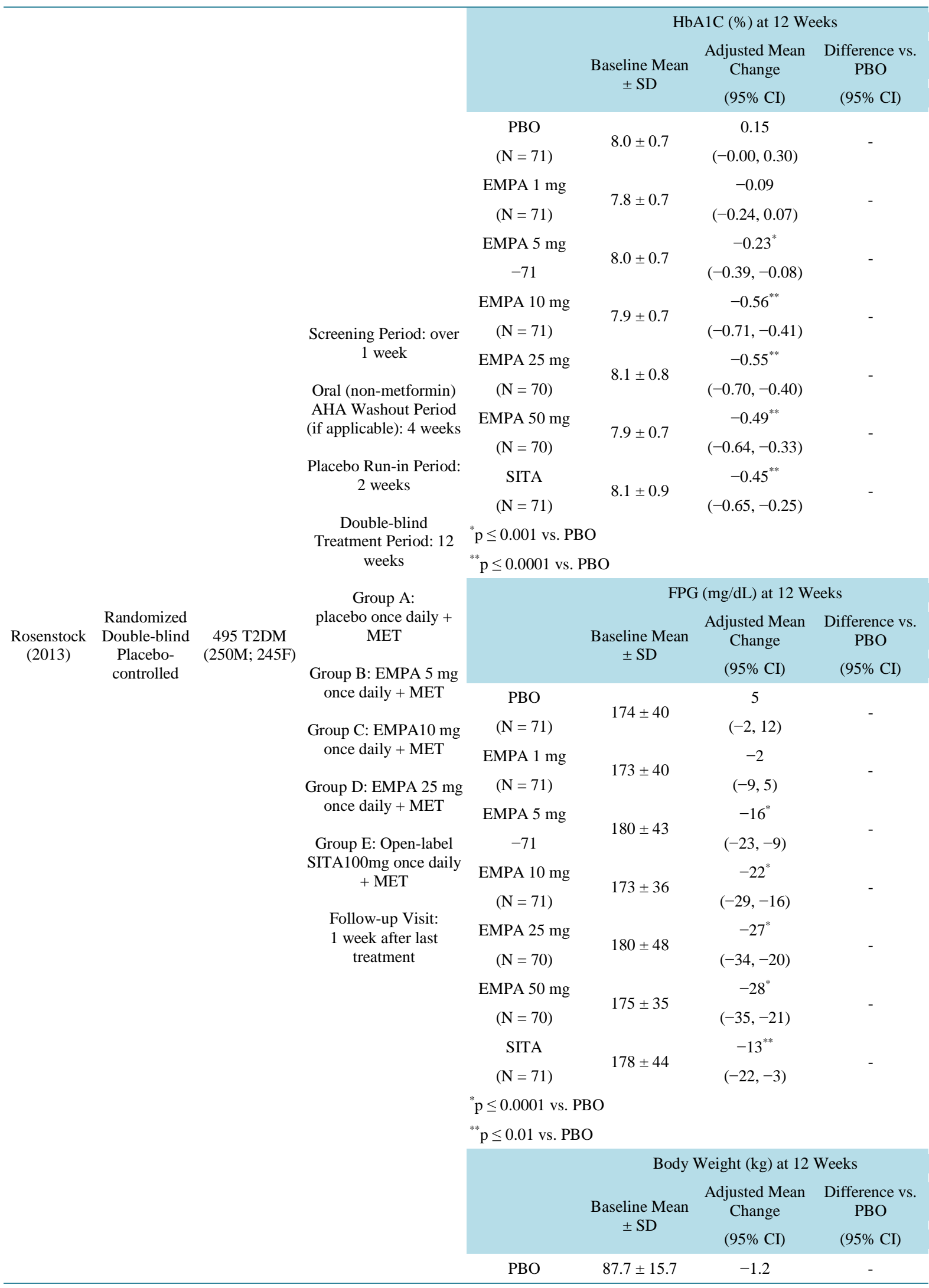




\section{Continued}

\begin{tabular}{|c|c|c|c|}
\hline$(\mathrm{N}=71)$ & & $(-1.8,-0.5)$ & \\
\hline $\begin{array}{l}\text { EMPA } 1 \mathrm{mg} \\
\qquad(\mathrm{N}=71)\end{array}$ & $90.6 \pm 18.9$ & $\begin{array}{c}-1.6 \\
(-2.2,-0.9)\end{array}$ & - \\
\hline $\begin{array}{l}\text { EMPA } 5 \mathrm{mg} \\
\quad-71\end{array}$ & $87.0 \pm 14.8$ & $\begin{array}{c}-2.3^{*} \\
(-2.9,-1.7)\end{array}$ & - \\
\hline $\begin{array}{l}\text { EMPA } 10 \mathrm{mg} \\
\qquad(\mathrm{N}=71)\end{array}$ & $87.9 \pm 14.4$ & $\begin{array}{c}-2.7^{* *} \\
(-3.4,-2.1)\end{array}$ & - \\
\hline $\begin{array}{l}\text { EMPA } 25 \text { mg } \\
\qquad(\mathrm{N}=70)\end{array}$ & $90.5 \pm 16.9$ & $\begin{array}{c}-2.6^{* *} \\
(-3.2,-2.0)\end{array}$ & - \\
\hline $\begin{array}{l}\text { EMPA } 50 \mathrm{mg} \\
\qquad(\mathrm{N}=70)\end{array}$ & $91.6 \pm 15.8$ & $\begin{array}{c}-2.9^{* * *} \\
(-3.5,-2.2)\end{array}$ & - \\
\hline $\begin{array}{c}\text { SITA } \\
(\mathrm{N}=71)\end{array}$ & $88.0 \pm 15.0$ & $\begin{array}{c}-0.8 \\
(-1.5,-0.2)\end{array}$ & - \\
\hline \multicolumn{4}{|c|}{${ }^{*} \mathrm{p} \leq 0.01$ vs. $\mathrm{PBO}$} \\
\hline \multicolumn{4}{|c|}{${ }^{* *} \mathrm{p} \leq 0.001$ vs. $\mathrm{PBO}$} \\
\hline \multicolumn{3}{|c|}{${ }^{* * *} \mathrm{p} \leq 0.0001$ vs. $\mathrm{PBO}$} & \\
\hline
\end{tabular}

95\% CI = 95\% confidence interval; AHA = antihyperglycemic agent; EMPA = empagliflozin; $\mathrm{F}$ = Female; FPG = Fasting Plasma Glucose; HbA1C = hemoglobin A1C; M = Male; MET = metformin; PBO = placebo; SD = standard deviation; SITA = sitagliptin; T2DM = Type 2 Diabetes Mellitus Patients.

\section{Conclusion}

Considering the increasing prevalence and incidence of type 2 diabetes mellitus worldwide, there is an obvious need for effective therapeutic strategies to combat this chronic and progressive disease. The need for agents with novel mechanisms of action is becoming more and more crucial owing to the need for individualized glycemic targets and glucose-lowering therapies, concerning side effects of many current therapies, and the progressive $\beta$-cell function decline associated with T2DM. SGLT2 inhibitors offer this potential and recently approved canagliflozin, dapagliflozin, and empagliflozin have shown significant promise as mono- and add-on therapy to current glucose-lowering regimens that may not otherwise be providing sufficient glycemic control in T2DM patients. Short-term benefits have certainly been made clear through the variety of clinical trials performed on these drugs, however there is still a need to establish long-term safety and efficacy. The significance of the unique side effects of increased genital mycotic infections and associated adverse events must also be considered. Several other agents in this class are in phase III trials and show similar promise in their efficacy as addon treatments.

\section{Acknowledgements}

We acknowledge Jennifer Heo, Sarah Jaffery, and Malay Naik for editorial review.

\section{References}

[1] Centers for Disease Control and Prevention (2012) Diabetes Data \& Trends. Department of Health and Human Services, Atlanta. http://apps.nccd.cdc.gov/DDTSTRS/default.aspx

[2] (2014) Farxiga (Dapagliflozin) (Prescribing Information). Bristol-Myers Squibb Company, Princeton.

[3] (2014) Jardiance (Empagliflozin) (Prescribing Information). Boehringer Ingelheim Pharmaceuticals, Inc., Ridgefield.

[4] (2013) Invokana (Canagliflozin) (Prescribing Information). Janssen Pharmaceuticals, Titusville.

[5] Hardman, T.C. and Dubrey, S.W. (2011) Development and Potential Role of Type-2 Sodium-Glucose Transporter Inhibitors for Management of Type 2 Diabetes. Diabetes Therapy, 2, 133-145. http://dx.doi.org/10.1007/s13300-011-0004-1

[6] Freeman, J.S. (2013) Review of Insulin-Dependent and Insulin-Independent Agents for Treating Patients with Type 2 
Diabetes Mellitus and Potential Role for Sodium-Glucose Co-Transporter 2 Inhibitors. Postgraduate Medicine, 125, 214-226. http://dx.doi.org/10.3810/pgm.2013.05.2672

[7] Rosenstock, J., Aggarwal, N., Polidori, D., Zhao, Y., Arbit, D., Usiskin, K., et al. (2012) Dose-Ranging Effects of Canagliflozin, a Sodium-Glucose Cotransporter 2 Inhibitor, as Add-On to Metformin in Subjects with Type 2 Diabetes. Diabetes Care, 35, 1232-1238. http://dx.doi.org/10.2337/dc11-1926

[8] Bode, B., Stenlöf, K., Sullivan, D., Fung, A. and Usiskin, K. (2013) Efficacy and Safety of Canagliflozin Treatment in Older Subjects with Type 2 Diabetes Mellitus: A Randomized Trial. Hospital Practice, 41, 72-84. http://dx.doi.org/10.3810/hp.2013.04.1020

[9] Cefalu, W.T., Leiter, L.A., Yoon, K.H., Arias, P., Niskanen, L., Xie, J., et al. (2013) Efficacy and Safety of Canagliflozin versus Glimepiride in Patients with Type 2 Diabetes Inadequately Controlled with Metformin (CANTATA-SU): 52 Week Results from a Randomised, Double-Blind, Phase 3 Non-Inferiority Trial. The Lancet, 382, 941-950. http://dx.doi.org/10.1016/S0140-6736(13)60683-2

[10] Inagaki, N., Kondo, K., Yoshinari, T., Maruyama, N., Susuta, Y. and Kuki, H. (2013) Efficacy and Safety of Canagliflozin in Japanese Patients with Type 2 Diabetes: A Randomized, Double-Blind, Placebo-Controlled, 12-Week Study. Diabetes, Obesityand Metabolism, 15, 1136-1145. http://dx.doi.org/10.1111/dom.12149

[11] Lavalle-González, F.J., Januszewicz, A., Davidson, J., Tong, C., Qiu, R., Canovatchel, W., et al. (2013) Efficacy and Safety of Canagliflozin Compared with Placebo and Sitagliptin in Patients with Type 2 Diabetes on Background Metformin Monotherapy: A Randomised trial. Diabetologia, 56, 2582-2592. http://dx.doi.org/10.1007/s00125-013-3039-1

[12] Schernthaner, G., Gross, J.L., Rosenstock, J., Guarisco, M., Fu, M., Yee, J., et al. (2013) Canagliflozin Compared with Sitagliptin for Patients with Type 2 Diabetes Who Do Not Have Adequate Glycemic Control with Metformin Plus Sulfonylurea: A 52-Week Randomized Trial. Diabetes Care, 36, 2508-2515. http://dx.doi.org/10.2337/dc12-2491

[13] Stenlöf, K., Cefalu, W.T., Kim, K.A., Alba, M., Usiskin, K., Tong, C., et al. (2013) Efficacy and Safety of Canagliflozin Monotherapy in Subjects with Type 2 Diabetes Mellitus Inadequately Controlled with Diet and Exercise. Diabetes, Obesity and Metabolism, 15, 372-382. http://dx.doi.org/10.1111/dom.12054

[14] Wilding, J.P., Charpentier, G., Hollander, P., González-Gálvez, G., Mathieu, C., Vercruysse, F., et al. (2013) Efficacy and Safety of Canagliflozin in Patients with Type 2 Diabetes Mellitus Inadequately Controlled with Metformin and Sulphonylurea: A Randomised Trial. International Journal of Clinical Practice, 67, 1267-1282. http://dx.doi.org/10.1111/ijcp.12322

[15] Yale, J.F., Bakris, G., Cariou, B., Yue, D., David-Neto, E., Xi, L., et al. (2013) Efficacy and Safety of Canagliflozin in Subjects with Type 2 Diabetes and Chronic Kidney Disease. Diabetes, Obesity and Metabolism, 15, 463-473. http://dx.doi.org/10.1111/dom.12090

[16] Forst, T., Guthrie, R., Goldenberg, R., Yee, J., Vijapurkar, U., Meininger, G., et al. (2014) Efficacy and Safety of Canagliflozin over 52 Weeks in Patients with Type 2 Diabetes on Background Metformin and Pioglitazone. Diabetes, Obesity Metabolism, 16, 467-477. http://dx.doi.org/10.1111/dom.12273

[17] Burki, T.K. (2012) FDA Rejects Novel Diabetes Drug over Safety Fears. The Lancet, 379, 507. http://dx.doi.org/10.1016/S0140-6736(12)60216-5

[18] Bailey, C.J., Gross, J.L., Pieters, A., Bastien, A. and List, J.F. (2010) Effect of Dapagliflozin in Patients with Type 2 Diabetes Who Have Inadequate Glycaemic Control with Metformin: A Randomised, Double-Blind, Placebo-Controlled Trial. The Lancet, 375, 2223-2233. http://dx.doi.org/10.1016/S0140-6736(10)60407-2

[19] Bailey, C.J., Iqbal, N., T’joen, C. and List, J.F. (2012) Dapagliflozin Monotherapy in Drug-Naïve Patients with Diabetes: A Randomized-Controlled Trial of Low-Dose Range. Diabetes, Obesity and Metabolism, 14, 951-959. http://dx.doi.org/10.1111/j.1463-1326.2012.01659.x

[20] Bailey, C.J., Gross, J.L., Hennicken, D., Iqbal, N., Mansfield, T.A. and List, J.F. (2013) Dapagliflozin Add-On to Metformin in Type 2 Diabetes Inadequately Controlled with Metformin: A Randomized, Double-Blind, Placebo-Controlled 102-Week Trial. BMC Medicine, 11, 43. http://dx.doi.org/10.1186/1741-7015-11-43

[21] Ferrannini, E., Ramos, S.J., Salsali, A., Tang, W. and List, J.F. (2010) Dapagliflozin Monotherapy in Type 2 Diabetic Patients with Inadequate Glycemic Control by Diet and Exercise: A Randomized, Double-Blind, Placebo-Controlled, Phase 3 Trial. Diabetes Care, 33, 2217-2224. http://dx.doi.org/10.2337/dc10-0612

[22] Henry, R.R., Murray, A.V., Marmolejo, M.H., Hennicken, D., Ptaszynska, A. and List, J.F. (2012) Dapagliflozin, Metformin XR, or Both: Initial Pharmacotherapy for Type 2 Diabetes, a Randomised Controlled Trial. International Journal of Clinical Practice, 66, 446-456. http://dx.doi.org/10.1111/j.1742-1241.2012.02911.x

[23] Kaku, K., Inoue, S., Matsuoka, O., Kiyosue, A., Azuma, H., Hayashi, N., et al. (2013) Efficacy and Safety of Dapagliflozin as a Monotherapy for Type 2 Diabetes Mellitus in Japanese Patients with Inadequate Glycaemic Control: A Phase II Multicentre, Randomized, Double-Blind, Placebo-Controlled Trial. Diabetes, Obesity and Metabolism, 15, 432-440. http://dx.doi.org/10.1111/dom.12047 
[24] Nauck, M.A., Del Prato, S., Meier, J.J., Durán-García, S., Rohwedder, K., Elze, M., et al. (2011) Dapagliflozin versus Glipizide as Add-On Therapy in Patients with Type 2 Diabetes Who Have Inadequate Glycemic Control with Metformin: A Randomized, 52-Week, Double-Blind, Active-Controlled Noninferiority Trial. Diabetes Care, 34, $2015-2022$. http://dx.doi.org/10.2337/dc11-0606

[25] Rosenstock, J., Vico, M., Wei, L., Salsali, A. and List, J.F. (2012) Effects of Dapagliflozin, an SGLT2 Inhibitor, on $\mathrm{HbA}_{1 \mathrm{c}}$, Body Weight, and Hypoglycemia Risk in Patients with Type 2 Diabetes Inadequately Controlled on Pioglitazone Monotherapy. Diabetes Care, 35, 1473-1478. http://dx.doi.org/10.2337/dc11-1693

[26] Strojek, K., Yoon, K.H., Hruba, V., Elze, M., Langkilde, A.M. and Parikh, S. (2011) Effect of Dapagliflozin in Patients with Type 2 Diabetes Who Have Inadequate Glycaemic Control with Glimepiride: A Randomized, 24-Week, DoubleBlind, Placebo-Controlled Trial. Diabetes, Obesity and Metabolism, 13, 928-938. http://dx.doi.org/10.1111/j.1463-1326.2011.01434.x

[27] Wilding, J.P., Norwood, P., Tjoen, C., Bastien, A., List, J.F. and Fiedorek, F.T. (2009) A Study of Dapagliflozin in Patients with Type 2 Diabetes Receiving High Doses of Insulin Plus Insulin Sensitizers: Applicability of a Novel InsulinIndependent Treatment. Diabetes Care, 32, 1656-1662. http://dx.doi.org/10.2337/dc09-0517

[28] Wilding, J.P., Woo, V., Soler, N.G., Pahor, A., Sugg, J., Rohwedder, K., et al. (2012) Long-Term Efficacy of Dapagliflozin in Patients with Type 2 Diabetes Mellitus Receiving High Doses of Insulin: A Randomized Trial. Annals of Internal Medicine, 156, 405-415. http://dx.doi.org/10.7326/0003-4819-156-6-201203200-00003

[29] Heise, T., Seewaldt-Becker, E., Macha, S., Hantel, S., Pinnetti, S., Seman, L., et al. (2013) Safety, Tolerability, Pharmacokinetics and Pharmacodynamics Following 4 Weeks' Treatment with Empagliflozin Once Daily in Patients with Type 2 Diabetes. Diabetes, Obesity and Metabolism, 15, 613-621. http://dx.doi.org/10.1111/dom.12073

[30] Ferrannini, E., Seman, L., Seewaldt-Becker, E., Hantel, S., Pinnetti, S. and Woerle, H.J. (2013) A Phase IIb, Randomized, Placebo-Controlled Study of the SGLT2 Inhibitor Empagliflozin in Patients with Type 2 Diabetes. Diabetes, Obesity and Metabolism, 15, 721-728. http://dx.doi.org/10.1111/dom.12081

[31] Rosenstock, J., Seman, L.J., Jelaska, A., Hantel, S., Pinnetti, S., Hach, T., et al. (2013) Efficacy and Safety of Empagliflozin, a Sodium Glucose Cotransporter 2 (SGLT2) Inhibitor, as Add-On to Metformin in Type 2 Diabetes with Mild Hyperglycaemia. Diabetes, Obesity and Metabolism, 15, 1154-1160. http://dx.doi.org/10.1111/dom.12185 
Scientific Research Publishing (SCIRP) is one of the largest Open Access journal publishers. It is currently publishing more than 200 open access, online, peer-reviewed journals covering a wide range of academic disciplines. SCIRP serves the worldwide academic communities and contributes to the progress and application of science with its publication.

Other selected journals from SCIRP are listed as below. Submit your manuscript to us via either submit@scirp.org or Online Submission Portal.
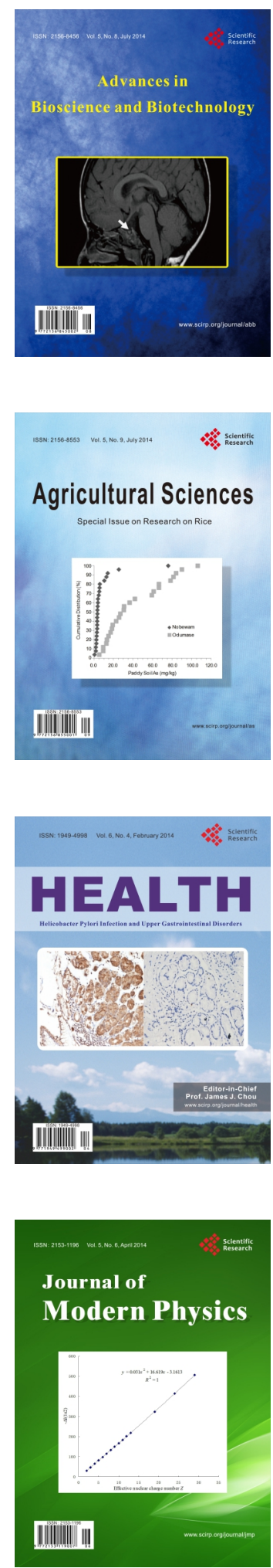
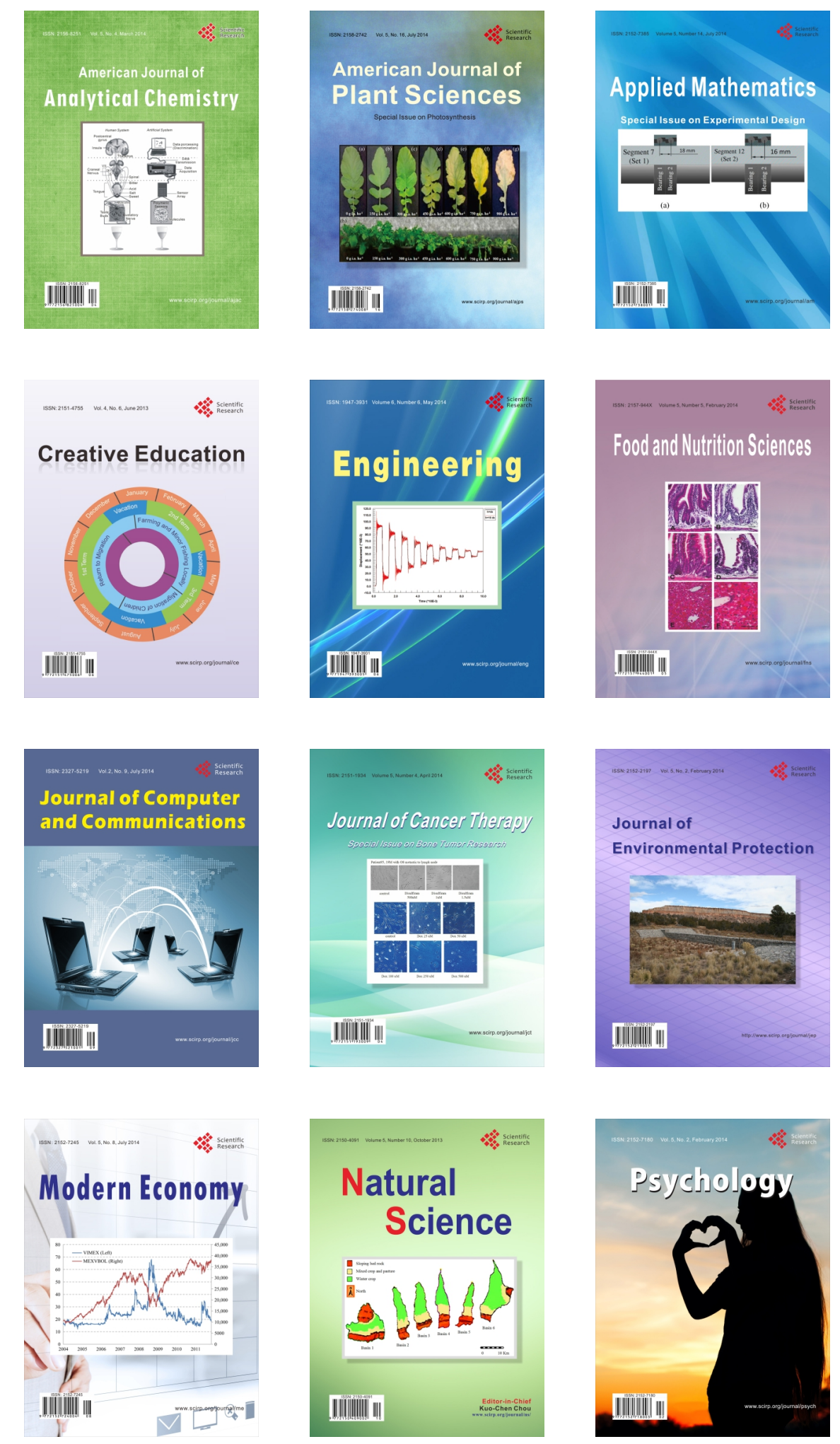